\title{
Elliptic determinant evaluations and the Macdonald identities for affine root systems
}

\author{
Hjalmar Rosengren and Michael Schlosser
}

\begin{abstract}
We obtain several determinant evaluations, related to affine root systems, which provide elliptic extensions of Weyl denominator formulas. Some of these are new, also in the polynomial special case, while others yield new proofs of the Macdonald identities for the seven infinite families of irreducible reduced affine root systems.
\end{abstract}

\section{Introduction}

Determinants play an important role in many areas of mathematics. Often, the solution of a particular problem in combinatorics, mathematical physics or, simply, linear algebra, depends on the explicit computation of a determinant. Some useful and efficient tools for evaluating determinants are provided in Krattenthaler's survey articles [Kra99, Kra05], which also contain many explicit determinant evaluations that have appeared in the literature and give references where further such formulas can be found.

As examples of interesting determinant evaluations, we mention the Weyl denominator formulas for classical root systems, which play a fundamental role in Lie theory and related areas. In general, the Weyl denominator formula for a reduced root system reads

$$
\sum_{w \in W} \operatorname{det}(w) e^{w(\rho)-\rho}=\prod_{\alpha \in R_{+}}\left(1-e^{-\alpha}\right),
$$

where $W$ is the Weyl group, $R_{+}$the set of positive roots and $\rho=\frac{1}{2} \sum_{\alpha \in R_{+}} \alpha$. For the classical root systems $A_{n-1}, B_{n}, C_{n}$ and $D_{n}$, this identity takes the explicit form

$$
\begin{aligned}
\operatorname{det}_{1 \leqslant i, j \leqslant n}\left(x_{i}^{j-1}\right) & =\prod_{1 \leqslant i<j \leqslant n}\left(x_{j}-x_{i}\right), \\
\operatorname{det}_{1 \leqslant i, j \leqslant n}\left(x_{i}^{j-n}-x_{i}^{n+1-j}\right) & =\prod_{i=1}^{n} x_{i}^{1-n}\left(1-x_{i}\right) \prod_{1 \leqslant i<j \leqslant n}\left(x_{j}-x_{i}\right)\left(1-x_{i} x_{j}\right), \\
\operatorname{dett}_{1 \leqslant i, j \leqslant n}\left(x_{i}^{j-n-1}-x_{i}^{n+1-j}\right) & =\prod_{i=1}^{n} x_{i}^{-n}\left(1-x_{i}^{2}\right) \prod_{1 \leqslant i<j \leqslant n}\left(x_{j}-x_{i}\right)\left(1-x_{i} x_{j}\right), \\
\operatorname{det}_{1 \leqslant i, j \leqslant n}\left(x_{i}^{j-n}+x_{i}^{n-j}\right) & =2 \prod_{i=1}^{n} x_{i}^{1-n} \prod_{1 \leqslant i<j \leqslant n}\left(x_{j}-x_{i}\right)\left(1-x_{i} x_{j}\right),
\end{aligned}
$$

respectively.

Received 11 May 2005, accepted in final form 21 October 2005.

2000 Mathematics Subject Classification 15A15 (primary), 17B67, 33E05 (secondary).

Keywords: determinants, theta functions, elliptic functions, affine root systems, Weyl denominator formula, Macdonald identities.

Research partially supported by EC's IHRP Programme, grant HPRN-CT-2001-00272 'Algebraic Combinatorics in Europe'. The first author was supported by the Swedish Science Research Council (VR). The second author was fully supported by FWF Austrian Science Fund grant P17563-N13.

This journal is (c) Foundation Compositio Mathematica 2006. 


\section{H. Rosengren And M. Schlosser}

In this article, we are interested in generalizing (1.2) to the level of elliptic determinant evaluations. By this we mean that the matrix elements should be defined in terms of theta functions, so that it is a priori clear that the quotient of the two sides of the identity is an elliptic function of some natural parameters. Up to date, according to our knowledge, very few elliptic determinant (and pfaffian) evaluations are known, see [Fro82], [FS77], [Has97, Lemma 1], [Oka04], [Rai05, Theorem 2.10], [TV97, Appendix B] and [War02, Theorem 4.17, Lemma 5.3]. Most of these results contain elliptic extensions of Weyl denominators, and are thus apparently related to root systems.

An elliptic extension of the Weyl denominator formula was obtained by Macdonald [Mac72], see also [Dys72]. He introduced, and completely classified, affine root systems. Moreover, he extended the Weyl denominator formula to the case of reduced affine root systems. In this setting, both the root system and the Weyl group are infinite, so the resulting Macdonald identities equate an infinite series and an infinite product. The precise statement is more complicated than (1.1), see [Mac72, Theorem 8.1] and, for the special cases of interest to us, Corollary 6.2 below. The Macdonald identities can be interpreted in terms of Kac-Moody algebras [Kac90]. Notable special cases include Watson's quintuple product identity [Wat29] (for the affine root system $B C_{1}$ ), Winquist's identity [Win69] (for $B_{2}$ ) and the so-called septuple product identity [FK99, Hir83, Hir00] (put $x_{2}=-1$ in the $B C_{2}$ case of Proposition 6.1 below).

There are seven infinite families of irreducible reduced affine root systems and seven exceptional cases. We only consider the infinite families, which Macdonald denotes by $A, B, B^{\vee}, C, C^{\vee}, B C$ and $D$. They should not be confused with the classical root systems mentioned above. (For instance, the classical root system $B C_{n}$ is non-reduced whereas the affine root system $B C_{n}$ is reduced.) Although the corresponding Macdonald identities do give elliptic extensions of (1.2), it is only for type $C, C^{\vee}$ and $B C$ that they can immediately be written as determinant evaluations. Nevertheless, one of our goals is to rewrite all seven cases in determinant form, and prove them by an 'identification of factors' argument similar to the usual proof of the Vandermonde determinant (1.2a). This new proof of the Macdonald identities is rather similar to Stanton's elementary proof [Sta89], but the use of determinants makes the details more streamlined.

For each affine root system $R$ under consideration, we define a corresponding notion of an $R$ theta function. We then give a 'master determinant formula', Proposition 3.4, which expresses a determinant of $R$ theta functions as a constant times the $R$ Macdonald denominator. When the constant can be explicitly determined, we have a genuine determinant evaluation. Such explicit instances of the master formula include a determinant of Warnaar (Proposition 4.1 below), new generalized Weyl denominator formulas for all seven families of reduced affine root systems (Theorems 4.4 and 4.9 and Corollaries 4.11-4.15) and determinant versions of the Macdonald identities (Proposition 6.1). Theorem 4.4 includes as special cases the determinants of Frobenius and Hasegawa cited above, and has a non-trivial overlap with the determinant of Tarasov and Varchenko.

The most striking difference between our new elliptic denominator formulas and those found by Macdonald is the large number of free parameters in our identities. This probably makes the results more difficult to interpret in terms of, say, affine Lie algebras. On the other hand, the presence of free parameters seems useful for certain applications. Indeed, special cases of our identities have found applications to multidimensional basic and elliptic hypergeometric series and integrals, see [GK97, KN03, Rai03, Rai05, Ros01, Ros04, RS03, Sch97, Sch99, Sch00a, Sch00b, Spi03, War02], to the study of Ruijsenaars operators and related integrable systems [Has97, Rui87], to combinatorics, see [Kra99] for an extensive list of references, as well as to number theory [Ros05]. It thus seems very likely that our new results will find similar applications.

Our paper is organized as follows. Section 2 contains preliminaries on Jacobi theta functions. In $\S 3$ we introduce theta functions associated to the seven families of reduced affine root systems. We then give our master formula, Proposition 3.4. In $\S 4$ we obtain several elliptic determinant 


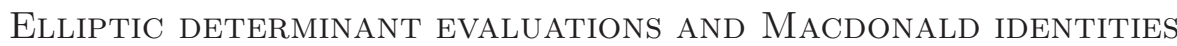

evaluations that can be viewed as explicit versions of Proposition 3.4. The main results are Theorems 4.4 and 4.9 (the other determinant evaluations are corollaries of these). Section 5 features several corollaries obtained by restricting to the polynomial special case. Finally, in $\S 6$, we obtain determinant evaluations that are shown to be equivalent to the Macdonald identities for non-exceptional reduced affine root systems.

\section{Preliminaries}

Throughout this paper, we implicitly assume that all scalars are generic, so that no denominators in our identities vanish.

The letter $p$ will denote a fixed number such that $0<|p|<1$. When dealing with the root system $C_{n}^{\vee}$, we will also assume a fixed choice of square root $p^{1 / 2}$. The case $p=0$ will be considered in $\S 5$.

We use the standard notation

$$
\begin{gathered}
(a)_{\infty}=(a ; p)_{\infty}=\prod_{j=0}^{\infty}\left(1-a p^{j}\right), \\
\left(a_{1}, \ldots, a_{n}\right)_{\infty}=\left(a_{1}, \ldots, a_{n} ; p\right)_{\infty}=\left(a_{1} ; p\right)_{\infty} \cdots\left(a_{n} ; p\right)_{\infty} .
\end{gathered}
$$

Then,

$$
\left(x^{k} ; p^{k}\right)_{\infty}=\prod_{j=0}^{k-1}\left(x \omega_{k}^{j} ; p\right)_{\infty}, \quad(x ; p)_{\infty}=\prod_{j=0}^{k-1}\left(x p^{j} ; p^{k}\right)_{\infty},
$$

where $\omega_{k}$ denotes a primitive $k$ th root of unity.

We employ 'multiplicative', rather than 'additive', notation for theta functions. This corresponds to realizing the torus $\mathbb{C} /(\mathbb{Z}+\tau \mathbb{Z})$ as $(\mathbb{C} \backslash\{0\}) /(z \mapsto p z)$, where $p=e^{2 \pi i \tau}$. Thus, we take as our building block the function

$$
\theta(x)=\theta(x ; p)=(x, p / x ; p)_{\infty} .
$$

We sometimes use the shorthand notation

$$
\begin{gathered}
\theta\left(a_{1}, \ldots, a_{n}\right)=\theta\left(a_{1}\right) \cdots \theta\left(a_{n}\right), \\
\theta\left(x y^{ \pm}\right)=\theta(x y) \theta(x / y) .
\end{gathered}
$$

The function $\theta(x)$ is holomorphic for $x \neq 0$ and has single zeroes precisely at $p^{\mathbb{Z}}$. Up to an elementary factor, $\theta\left(e^{2 \pi i x} ; e^{2 \pi i \tau}\right)$ equals the Jacobi theta function $\theta_{1}(x \mid \tau)$. We frequently use the inversion formula

and the quasi-periodicity

$$
\theta(1 / x)=-\frac{1}{x} \theta(x)
$$

$$
\theta(p x)=-\frac{1}{x} \theta(x)
$$

By Jacobi's triple product identity, we have the Laurent expansion

$$
\theta(x)=\frac{1}{(p)_{\infty}} \sum_{n=-\infty}^{\infty}(-1)^{n} p^{\left(\begin{array}{c}
n \\
2
\end{array}\right)} x^{n} .
$$

Similarly to (2.1), we have

$$
\theta\left(x^{k} ; p^{k}\right)=\prod_{j=0}^{k-1} \theta\left(x \omega_{k}^{j} ; p\right), \quad \theta(x ; p)=\prod_{j=0}^{k-1} \theta\left(x p^{j} ; p^{k}\right),
$$




\section{H. Rosengren And M. Schlosser}

which, when $k=2$, implies that

$$
\theta\left(x^{2}\right)=\theta\left(x,-x, p^{\frac{1}{2}} x,-p^{\frac{1}{2}} x\right) .
$$

Since $\theta(x)$ has a single zero at $x=1$, it follows that

$$
\theta\left(-1, p^{\frac{1}{2}},-p^{\frac{1}{2}}\right)=\lim _{x \rightarrow 1} \frac{\theta\left(x^{2}\right)}{\theta(x)}=2 .
$$

\section{Theta functions on root systems}

The Macdonald identities involve the Macdonald denominator

$$
\prod_{\alpha \in R_{+}}\left(1-e^{-\alpha}\right)
$$

where $R_{+}$is the positive part of a reduced affine root system and $e^{\alpha}$ a formal exponential. Although we will not need anything of Macdonald's theory, it may be instructive to explain what (3.1) means in the case $R=C_{n}$. Let $e_{i}, 1 \leqslant i \leqslant n$, be a basis for $\mathbb{R}^{n}$, and write $k+\varepsilon_{i}$ for the affine function $e_{j} \mapsto k+\delta_{i j}$. Then, affine $C_{n}$ consists of the roots

$$
\begin{gathered}
k \pm 2 \varepsilon_{i}, \quad k \in \mathbb{Z}, 1 \leqslant i \leqslant n, \\
k \pm \varepsilon_{i} \pm \varepsilon_{j}, \quad k \in \mathbb{Z}, 1 \leqslant i<j \leqslant n .
\end{gathered}
$$

The positive roots are

$$
\begin{gathered}
k+2 \varepsilon_{i}, \quad k \geqslant 0, \quad 1 \leqslant i \leqslant n, \\
k-2 \varepsilon_{i}, \quad k \geqslant 1, \quad 1 \leqslant i \leqslant n, \\
k+\varepsilon_{i}+\varepsilon_{j}, \quad k+\varepsilon_{i}-\varepsilon_{j}, \quad k \geqslant 0,1 \leqslant i<j \leqslant n, \\
k-\varepsilon_{i}+\varepsilon_{j}, \quad k-\varepsilon_{i}-\varepsilon_{j}, \quad k \geqslant 1, \quad 1 \leqslant i<j \leqslant n .
\end{gathered}
$$

Thus, the Macdonald denominator for $C_{n}$ is

$$
\begin{aligned}
& \prod_{i=1}^{n} \prod_{k=0}^{\infty}\left(1-e^{-\left(k+2 \varepsilon_{i}\right)}\right)\left(1-e^{-\left(k+1-2 \varepsilon_{i}\right)}\right) \\
& \quad \times \quad \prod_{1 \leqslant i<j \leqslant n} \prod_{k=0}^{\infty}\left(1-e^{-\left(k+\varepsilon_{i}+\varepsilon_{j}\right)}\right)\left(1-e^{-\left(k+\varepsilon_{i}-\varepsilon_{j}\right)}\right)\left(1-e^{-\left(k+1-\varepsilon_{i}+\varepsilon_{j}\right)}\right)\left(1-e^{-\left(k+1-\varepsilon_{i}-\varepsilon_{j}\right)}\right) .
\end{aligned}
$$

Introducing variables $p$ and $x_{1}, \ldots, x_{n}$ by $p=e^{-1}, x_{i}=p^{-\varepsilon_{i}},(3.2)$ takes the form

$$
\prod_{i=1}^{n} \theta\left(x_{i}^{2}\right) \prod_{1 \leqslant i<j \leqslant n} \theta\left(x_{i} x_{j}^{ \pm}\right),
$$

where $\theta(x)=\theta(x ; p)$. The $C_{n}$ Macdonald identity gives the explicit multiple Laurent expansion of this function, where $x_{i}$ are viewed as non-zero complex variables and $p$ as a constant with $|p|<1$.

More generally, the Macdonald denominators for the seven families of reduced affine root systems equal, up to a trivial factor that has been chosen for convenience, 
Elliptic Determinant eVAluations And Macdonald identities

$$
\begin{aligned}
W_{A_{n-1}}(x) & =\prod_{1 \leqslant i<j \leqslant n} x_{j} \theta\left(x_{i} / x_{j}\right), \\
W_{B_{n}}(x) & =\prod_{i=1}^{n} \theta\left(x_{i}\right) \prod_{1 \leqslant i<j \leqslant n} x_{i}^{-1} \theta\left(x_{i} x_{j}^{ \pm}\right), \\
W_{B_{n}^{\vee}}(x) & =\prod_{i=1}^{n} x_{i}^{-1} \theta\left(x_{i}^{2} ; p^{2}\right) \prod_{1 \leqslant i<j \leqslant n} x_{i}^{-1} \theta\left(x_{i} x_{j}^{ \pm}\right), \\
W_{C_{n}}(x) & =\prod_{i=1}^{n} x_{i}^{-1} \theta\left(x_{i}^{2}\right) \prod_{1 \leqslant i<j \leqslant n} x_{i}^{-1} \theta\left(x_{i} x_{j}^{ \pm}\right), \\
W_{C_{n}^{\vee}}(x) & =\prod_{i=1}^{n} \theta\left(x_{i} ; p^{\frac{1}{2}}\right) \prod_{1 \leqslant i<j \leqslant n} x_{i}^{-1} \theta\left(x_{i} x_{j}^{ \pm}\right), \\
W_{B C_{n}}(x) & =\prod_{i=1}^{n} \theta\left(x_{i}\right) \theta\left(p x_{i}^{2} ; p^{2}\right) \prod_{1 \leqslant i<j \leqslant n} x_{i}^{-1} \theta\left(x_{i} x_{j}^{ \pm}\right), \\
W_{D_{n}}(x) & =\prod_{1 \leqslant i<j \leqslant n} x_{i}^{-1} \theta\left(x_{i} x_{j}^{ \pm}\right) .
\end{aligned}
$$

We use the above list as a rule for labelling our results. Each of our elliptic determinant evaluations expresses the Macdonald denominator of some affine root system as a determinant.

The following definition may seem strange, since root systems are usually associated to multivariable functions. However, it will enable us to give a very succinct statement of Proposition 3.4. Note that, except in the case $R=A_{n-1}, W_{R}$ is an $R$ theta function of each $x_{i}$. This is easy to check directly, and is also clear from Proposition 3.4.

Definition 3.1. Let $f(x)$ be holomorphic for $x \neq 0$. Then, we call $f$ an $A_{n-1}$ theta function of norm $t$ if

$$
f(p x)=\frac{(-1)^{n}}{t x^{n}} f(x)
$$

Moreover, if $R$ denotes $B_{n}, B_{n}^{\vee}, C_{n}, C_{n}^{\vee}, B C_{n}$ or $D_{n}$, we call $f$ an $R$ theta function if

$$
\begin{aligned}
& f(p x)=-\frac{1}{p^{n-1} x^{2 n-1}} f(x), \quad f(1 / x)=-\frac{1}{x} f(x), \quad R=B_{n}, \\
& f(p x)=-\frac{1}{p^{n} x^{2 n}} f(x), \quad f(1 / x)=-f(x), \quad R=B_{n}^{\vee}, \\
& f(p x)=\frac{1}{p^{n+1} x^{2 n+2}} f(x), \quad f(1 / x)=-f(x), \quad R=C_{n}, \\
& f(p x)=\frac{1}{p^{n-\frac{1}{2}} x^{2 n}} f(x), \quad f(1 / x)=-\frac{1}{x} f(x), \quad R=C_{n}^{\vee}, \\
& f(p x)=\frac{1}{p^{n} x^{2 n+1}} f(x), \quad f(1 / x)=-\frac{1}{x} f(x), \quad R=B C_{n}, \\
& f(p x)=\frac{1}{p^{n-1} x^{2 n-2}} f(x), \quad f(1 / x)=f(x), \quad R=D_{n} .
\end{aligned}
$$
$p^{1 / 2}$.

These notions depend on our fixed parameter $p$, and in the case of $C_{n}^{\vee}$ on a choice of square root

The following result gives useful factorizations of $R$ theta functions. 


\section{H. Rosengren And M. Schlosser}

Lemma 3.2. The function $f$ is an $A_{n-1}$ theta function of norm $t$ if and only if there exist constants $C, b_{1}, \ldots, b_{n}$ such that $b_{1} \cdots b_{n}=t$ and

$$
f(x)=C \theta\left(b_{1} x, \ldots, b_{n} x\right) .
$$

For the other six cases, $f$ is an $R$ theta function if and only if there exist constants $C, b_{1}, \ldots, b_{n-1}$ such that

$$
\begin{aligned}
& f(x)=C \theta(x) \theta\left(b_{1} x^{ \pm}, \ldots, b_{n-1} x^{ \pm}\right), \quad R=B_{n}, \\
& f(x)=C x^{-1} \theta\left(x^{2} ; p^{2}\right) \theta\left(b_{1} x^{ \pm}, \ldots, b_{n-1} x^{ \pm}\right), \quad R=B_{n}^{\vee}, \\
& f(x)=C x^{-1} \theta\left(x^{2}\right) \theta\left(b_{1} x^{ \pm}, \ldots, b_{n-1} x^{ \pm}\right), \quad R=C_{n}, \\
& f(x)=C \theta\left(x ; p^{\frac{1}{2}}\right) \theta\left(b_{1} x^{ \pm}, \ldots, b_{n-1} x^{ \pm}\right), \quad R=C_{n}^{\vee}, \\
& f(x)=C \theta(x) \theta\left(p x^{2} ; p^{2}\right) \theta\left(b_{1} x^{ \pm}, \ldots, b_{n-1} x^{ \pm}\right), \quad R=B C_{n}, \\
& f(x)=C \theta\left(b_{1} x^{ \pm}, \ldots, b_{n-1} x^{ \pm}\right), \quad R=D_{n},
\end{aligned}
$$

where $\theta(x)=\theta(x ; p)$.

Proof. Up to the change of variable $x \mapsto e^{2 \pi i x}$, what we call an $A_{n-1}$ theta function is usually called a theta function of order $n$. In that case, the factorization theorem is classical, see [Web91, p. 45]. Nevertheless, we review the proof. The 'if' part is straightforward, so we assume that $f$ is an $A_{n-1}$ theta function. Let $N$ be the number of zeroes of $f$, counted with multiplicity, inside any period annulus $A=\{|p| r<|x| \leqslant r\}$. It is well-known that

$$
N=\int_{\partial A} \frac{f^{\prime}(x)}{f(x)} \frac{d x}{2 \pi i}
$$

The equality (3.3) differentiates to

$$
\frac{f^{\prime}(x)}{f(x)}-p \frac{f^{\prime}(p x)}{f(p x)}=\frac{n}{x}
$$

which gives $N=n$. Thus, there exist $b_{1}, \ldots, b_{n}$ so that the zeroes, counted with multiplicity, are enumerated by $p^{m} b_{i}, m \in \mathbb{Z}, i=1, \ldots, n$. The function $g(x)=f(x) / \theta\left(b_{1} x, \ldots, b_{n} x\right)$ is then analytic for $x \neq 0$ and satisfies $g(p x)=g(x)$, so by Liouville's theorem it is constant. Finally, if $f$ has norm $t$, one checks that $b_{1} \cdots b_{n}=t$.

Let us now consider the case $R=D_{n}$. Since any $D_{n}$ theta function $f$ is an $A_{2 n-3}$ theta function, it has $2 n-2$ zeroes in each period annulus. It is easy to check from the definition that if $a$ is a zero, then $1 / a$ is a zero of the same multiplicity, and if some zero should satisfy $a^{2} \in p^{\mathbb{Z}}$, then its multiplicity is even. Thus, there exist $a_{1}, \ldots, a_{n-1}$ so that the zeroes, with multiplicity, are enumerated by $p^{m} a_{i}^{ \pm}, m \in \mathbb{Z}, i=1, \ldots, n-1$. As before, $g(x)=f(x) / \theta\left(a_{1} x^{ \pm}, \ldots, a_{n-1} x^{ \pm}\right)$is analytic for $x \neq 0$ and satisfies $g(p x)=g(x)$, so by Liouville's theorem it is constant.

The other cases are easily deduced from the case $R=D_{n}$. For instance, assume that $f$ is a $B C_{n}$ theta function. Letting $x=1, x=1 / \sqrt{p}$ and $x=-1 / \sqrt{p}$ in Definition 3.1, one finds that $f$ vanishes at these points and thus $f\left(p^{m}\right)=f\left( \pm \sqrt{p} p^{m}\right)=0$ for any $m \in \mathbb{Z}$. It follows that $g(x)=f(x) / \theta(x) \theta\left(p x^{2} ; p^{2}\right)$ is analytic for $x \neq 0$. It is straightforward to check that $g$ is a $D_{n}$ theta function, so the desired factorization follows from the case $R=D_{n}$. The remaining cases can be treated similarly.

We also use the following result, which expresses $R$ theta functions, when $R$ is not of type $A$, in terms of type $A$ theta functions. 


\section{ELLiPtic DETERMinANT EVALUATIONS AND MACDONALD IDENTITIES}

Lemma 3.3. The function $f$ is an $R$ theta function if and only if there exists a function $g(x)$, holomorphic for $x \neq 0$, such that

$$
\begin{aligned}
& g(p x)=-\frac{1}{p^{n-1} x^{2 n-1}} g(x), \quad f(x)=g(x)-x g(1 / x), \quad R=B_{n}, \\
& g(p x)=-\frac{1}{p^{n} x^{2 n}} g(x), \quad f(x)=g(x)-g(1 / x), \quad R=B_{n}^{\vee}, \\
& g(p x)=\frac{1}{p^{n+1} x^{2 n+2}} g(x), \quad f(x)=g(x)-g(1 / x), \quad R=C_{n}, \\
& g(p x)=\frac{1}{p^{n-\frac{1}{2}} x^{2 n}} g(x), \quad f(x)=g(x)-x g(1 / x), \quad R=C_{n}^{\vee}, \\
& g(p x)=\frac{1}{p^{n} x^{2 n+1}} g(x), \quad f(x)=g(x)-x g(1 / x), \quad R=B C_{n}, \\
& g(p x)=\frac{1}{p^{n-1} x^{2 n-2}} g(x), \quad f(x)=g(x)+g(1 / x), \quad R=D_{n} .
\end{aligned}
$$

Proof. If $f$ is an $R$ theta function, one may in each case choose $g=f / 2$. The converse is straightforward.

An important example, to be used later, is the case when $R=C_{1}$ and $g(x)=x^{-2} \theta(a x, b x, c x, d x)$, $a b c d=1$. Combining Lemmas 3.2 and 3.3 gives $g(x)-g(1 / x)=C x^{-1} \theta\left(x^{2}\right)$, where $C$ may be computed by plugging in $x=a$. This leads to the identity

$$
\frac{1}{x^{2}} \theta(a x, b x, c x, d x)-x^{2} \theta(a / x, b / x, c / x, d / x)=\frac{1}{a x} \theta\left(a b, a c, a d, x^{2}\right), \quad a b c d=1,
$$

which is equivalent to Riemann's addition formula (cf. [WW96, p. 451, Example 5]).

We are now in a position to state our 'master formula'.

Proposition 3.4. Let $f_{1}, \ldots, f_{n}$ be $A_{n-1}$ theta functions of norm $t$. Then,

$$
\operatorname{det}_{1 \leqslant i, j \leqslant n}\left(f_{j}\left(x_{i}\right)\right)=C \theta\left(t x_{1} \cdots x_{n}\right) W_{A_{n-1}}(x)
$$

for some constant $C$. Moreover, if $R$ denotes $B_{n}, B_{n}^{\vee}, C_{n}, C_{n}^{\vee}, B C_{n}$ or $D_{n}$ and $f_{1}, \ldots, f_{n}$ are $R$ theta functions, we have

$$
\operatorname{det}_{1 \leqslant i, j \leqslant n}\left(f_{j}\left(x_{i}\right)\right)=C W_{R}(x)
$$

for some constant $C$.

Proof. Consider first the case of (3.5a). For fixed $i=1, \ldots, n$, let $L\left(x_{i}\right)$ and $R\left(x_{i}\right)$ denote the lefthand and right-hand sides, viewed as functions of $x_{i}$. It is straightforward to verify that both $L$ and $R$ are $A_{n-1}$ theta functions of norm $t$. Thus, $f=L / R$ satisfies $f(p x)=f(x)$, so if we can prove that $f$ is analytic, it follows from Liouville's theorem that it is constant. Up to multiplication with $p^{\mathbb{Z}}$, the zeroes of $R$ are situated at $x_{i}=x_{j}, j \neq i$ and at $x_{i}=1 / t x_{1} \cdots \hat{x}_{i} \cdots x_{n}$. For generic values of $x_{j}$, $j \neq i$, they are all single zeroes, so it is enough to show that $L$ vanishes at these points. In the first case, $x_{i}=x_{j}, j \neq i$, this is clear since the $i$ th and $j$ th rows in the determinant are equal. It then follows from Lemma 3.2 that $L$ vanishes also at $x_{i}=1 / t x_{1} \cdots \hat{x}_{i} \cdots x_{n}$.

In the other cases, the same proof works with obvious modifications. It is actually enough to go through this for $R=D_{n}$, since the remaining five cases can then be deduced using Lemma 3.2.

In the case $R=D_{n}$, one may well attribute Proposition 3.4 to Warnaar. Although he only states it in a special case, see Proposition 4.1 below, his proof extends verbatim to the general case. 


\section{H. Rosengren And M. Schlosser}

Remark 3.5. Replacing $x_{i}$ by $x_{i} / \sqrt[n]{t}$ one sees that $(3.5 \mathrm{a})$ is equivalent to its special case $t=1$. Thus, if we would redefine $W_{A_{n-1}}$ as $\theta\left(x_{1} \cdots x_{n}\right) W_{A_{n-1}}(x)$, we could give a unified statement of Proposition 3.4 for all root systems. We have chosen to formulate the result using the superfluous parameter $t$ since this seems convenient for applications, in particular to multidimensional hypergeometric series.

\section{Elliptic determinant evaluations}

We do not consider Proposition 3.4 as a determinant evaluation, since we do not have a simple formula for the constant $C$. From our perspective, the main use of Proposition 3.4 is to systematize our knowledge of elliptic determinant evaluations, as corresponding to various special cases when this constant can be computed.

\subsection{Warnaar's type $D$ determinant}

For comparison and completeness, we first review the following determinant evaluation due to Warnaar [War02, Lemma 5.3]. Warnaar used it to obtain a summation formula for a multidimensional elliptic hypergeometric series; further related applications may be found in [Ros01, Ros04, RS03, Spi03]. In the limit $p \rightarrow 0$ it reduces to Krattenthaler's determinant [Kra95, Lemma 34], which has been a powerful tool in the enumeration of, and computation of generating functions for, restricted families of plane partitions and tableaux, see the discussion of Lemmas 3-5 and Theorems 26-31 in [Kra99].

Warnaar's determinant corresponds to the case of Proposition 3.4 when $R=D_{n}$ and

$$
f_{j}(x)=P_{j}(x) \prod_{k=j+1}^{n} \theta\left(a_{k} x^{ \pm}\right),
$$

with $P_{j}$ a $D_{j}$ theta function. Then, for $x_{i}=a_{i}$, the matrix in (3.5b) is triangular, so that its determinant, and thus the constant $C$, can be computed. This leads to the following result.

Proposition 4.1 (A $D$ type determinant evaluation [War02]). Let $x_{1}, \ldots, x_{n}$ and $a_{1}, \ldots, a_{n}$ be indeterminates. For each $j=1, \ldots, n$, let $P_{j}$ be a $D_{j}$ theta function. Then there holds

$$
\operatorname{det}_{1 \leqslant i, j \leqslant n}\left(P_{j}\left(x_{i}\right) \prod_{k=j+1}^{n} \theta\left(a_{k} x_{i}^{ \pm}\right)\right)=\prod_{i=1}^{n} P_{i}\left(a_{i}\right) \prod_{1 \leqslant i<j \leqslant n} a_{j} x_{j}^{-1} \theta\left(x_{j} x_{i}^{ \pm}\right) .
$$

The parameter $a_{1}$ is introduced for convenience, its value being immaterial since $P_{1}$ is constant. Similar remarks can be made about many of our results below.

Corollary 4.2 (A $D$ type Cauchy determinant). Let $x_{1}, \ldots, x_{n}$ and $a_{1}, \ldots, a_{n}$ be indeterminates. Then there holds

$$
\operatorname{det}_{1 \leqslant i, j \leqslant n}\left(\frac{1}{\theta\left(a_{j} x_{i}^{ \pm}\right)}\right)=\frac{\prod_{1 \leqslant i<j \leqslant n} a_{j} x_{j}^{-1} \theta\left(x_{j} x_{i}^{ \pm}, a_{i} a_{j}^{ \pm}\right)}{\prod_{i, j=1}^{n} \theta\left(a_{j} x_{i}^{ \pm}\right)} .
$$

Proof. Let $P_{j}(x)=\prod_{k=1}^{j-1} \theta\left(a_{k} x^{ \pm}\right)$in Proposition 4.1, pull $\prod_{k=1}^{n} \theta\left(a_{k} x_{i}^{ \pm}\right)$out of the $i$ th row of the determinant $(i=1, \ldots, n)$ and divide both sides by $\prod_{i, j=1}^{n} \theta\left(a_{j} x_{i}^{ \pm}\right)$.

Corollary 4.2 was used by Rains [Rai03, Rai05] to obtain transformations and recurrences for multiple elliptic hypergeometric integrals. Perhaps surprisingly, it is equivalent to the classical Cauchy determinant

see [Rai05].

$$
\operatorname{det}_{1 \leqslant i, j \leqslant n}\left(\frac{1}{u_{i}+v_{j}}\right)=\frac{\prod_{1 \leqslant i<j \leqslant n}\left(u_{j}-u_{i}\right)\left(v_{j}-v_{i}\right)}{\prod_{i, j=1}^{n}\left(u_{i}+v_{j}\right)},
$$




\section{Elliptic DETERMinANT EVALUATIONS AND MACDONALD IDENTITIES}

Another simple consequence of Proposition 4.1 is the following determinant evaluation, which is included here for possible future reference. Two related determinant evaluations, corresponding to the type $A$ root system and restricted to the polynomial case, were applied in [Sch97, Sch00a] to obtain multidimensional matrix inversions that played a major role in the derivation of new summation formulae for multidimensional basic hypergeometric series, see Remark 5.4. Eventually, Corollary 4.3 may have similar applications in the elliptic setting.

Corollary 4.3 (A $D$ type determinant evaluation). Let $x_{1}, \ldots, x_{n}, a_{1}, \ldots, a_{n+1}$ and $b$ be indeterminates. For each $j=1, \ldots, n+1$, let $P_{j}$ be a $D_{j}$ theta function. Then there holds

$$
\begin{aligned}
P_{n+1}(b) & \operatorname{det}_{1 \leqslant i, j \leqslant n}\left(P_{j}\left(x_{i}\right) \prod_{k=j+1}^{n+1} \theta\left(a_{k} x_{i}^{ \pm}\right)-\frac{P_{n+1}\left(x_{i}\right)}{P_{n+1}(b)} P_{j}(b) \prod_{k=j+1}^{n+1} \theta\left(a_{k} b^{ \pm}\right)\right) \\
= & \prod_{i=1}^{n+1} P_{i}\left(a_{i}\right) \prod_{1 \leqslant i<j \leqslant n+1} a_{j} x_{j}^{-1} \theta\left(x_{j} x_{i}^{ \pm}\right),
\end{aligned}
$$

where $x_{n+1}=b$.

Proof. We proceed similarly as in the proof of Lemma A.1 of [Sch97]. In particular, we utilize $\operatorname{det}\left(\begin{array}{cc}M & \eta \\ \xi & \gamma\end{array}\right)=\gamma \operatorname{det}\left(M-\gamma^{-1} \eta \xi\right)$ (which is a special case of a formula due to Sylvester [Syl51]) applied to $M=\left(P_{j}\left(x_{i}\right) \prod_{k=j+1}^{n+1} \theta\left(a_{k} x_{i}^{ \pm}\right)\right), \xi=\left(P_{j}(b) \prod_{k=j+1}^{n+1} \theta\left(a_{k} b^{ \pm}\right)\right), \eta=\left(P_{n+1}\left(x_{i}\right)\right), \gamma=P_{n+1}(b)$, and then apply Proposition 4.1.

\subsection{An $A$ type determinant}

If one tries to imitate the proof of Proposition 4.1, using Proposition 3.4 for $B_{n}, B_{n}^{\vee}, C_{n}, C_{n}^{\vee}$ or $B C_{n}$, rather than $D_{n}$, one will find results that are equivalent to Proposition 4.1 in view of Lemma 3.2. However, for the root system $A_{n-1}$ one obtains the following new elliptic extension of the Vandermonde determinant (1.2a), see Remark 5.15.

Theorem 4.4 (An $A$ type determinant evaluation). Let $x_{1}, \ldots, x_{n}, a_{1}, \ldots, a_{n}$ and $t$ be indeterminates. For each $j=1, \ldots, n$, let $P_{j}$ be an $A_{j-1}$ theta function of norm $t a_{1} \cdots a_{j}$. Then there holds

$$
\operatorname{det}_{1 \leqslant i, j \leqslant n}\left(P_{j}\left(x_{i}\right) \prod_{k=j+1}^{n} \theta\left(a_{k} x_{i}\right)\right)=\frac{\theta\left(t a_{1} \cdots a_{n} x_{1} \cdots x_{n}\right)}{\theta(t)} \prod_{i=1}^{n} P_{i}\left(1 / a_{i}\right) \prod_{1 \leqslant i<j \leqslant n} a_{j} x_{j} \theta\left(x_{i} / x_{j}\right) .
$$

Proof. By the $A_{n-1}$ case of Proposition 3.4, with $t$ replaced by $t a_{1} \cdots a_{n}$, (4.1) holds up to a factor independent of $x_{i}$. To compute this constant one may let $x_{i}=1 / a_{i}$, in which case the matrix on the left-hand side is triangular.

By Lemma 3.2, we may without loss of generality assume that

$$
P_{j}(x)=\theta\left(b_{1 j} x\right) \cdots \theta\left(b_{j j} x\right),
$$

where $b_{1 j} \cdots b_{j j}=t a_{1} \cdots a_{j}$. On the right-hand side of $(4.1)$, we then have $P_{1}\left(1 / a_{1}\right) / \theta(t)=1$. After replacing $t$ by $t / a_{1} \cdots a_{n}$, this gives the following equivalent form of Theorem 4.4:

$$
\operatorname{det}_{1 \leqslant i, j \leqslant n}\left(\prod_{k=1}^{j} \theta\left(b_{k j} x_{i}\right) \prod_{k=j+1}^{n} \theta\left(a_{k} x_{i}\right)\right)=\theta\left(t x_{1} \cdots x_{n}\right) \prod_{i=2}^{n} \prod_{k=1}^{i} \theta\left(b_{k i} / a_{i}\right) \prod_{1 \leqslant i<j \leqslant n} a_{j} x_{j} \theta\left(x_{i} / x_{j}\right),
$$

where

$$
b_{1 j} \cdots b_{j j} a_{j+1} \cdots a_{n}=t, \quad j=1, \ldots, n .
$$

If we make the further specialization

$$
\left(b_{1 j}, \ldots, b_{j j}\right)=\left(c_{1}, \ldots, c_{j-1}, b_{j}\right)
$$




\section{H. Rosengren And M. Schlosser}

and then interchange $a_{j}$ and $c_{j}$, we recover the following determinant evaluation due to Tarasov and Varchenko. In a special case, it was also obtained by Hasegawa [Has97, Lemma 1], who used it to compute the trace of elliptic $L$-operators, leading to the elliptic Ruijsenaars(-Macdonald) commuting difference operators, see [Rui87].

Corollary 4.5 (Tarasov and Varchenko). Let $x_{1}, \ldots, x_{n}, a_{1}, \ldots, a_{n-1}, b_{1}, \ldots, b_{n}, c_{2}, \ldots, c_{n}$ and $t$ be indeterminates, such that

$$
a_{1} \cdots a_{j-1} b_{j} c_{j+1} \cdots c_{n}=t, \quad j=1, \ldots, n .
$$

Then there holds

$$
\operatorname{det}_{1 \leqslant i, j \leqslant n}\left(\prod_{k=1}^{j-1} \theta\left(a_{k} x_{i}\right) \cdot \theta\left(b_{j} x_{i}\right) \prod_{k=j+1}^{n} \theta\left(c_{k} x_{i}\right)\right)=\theta\left(t x_{1} \cdots x_{n}\right) \prod_{i=2}^{n} \theta\left(b_{i} / c_{i}\right) \prod_{1 \leqslant i<j \leqslant n} c_{j} x_{j} \theta\left(x_{i} / x_{j}, a_{i} / c_{j}\right) .
$$

Note that $\prod_{i=2}^{n} \theta\left(b_{i} / c_{i}\right)=\prod_{i=1}^{n-1} \theta\left(b_{i} / a_{i}\right)$.

Remark 4.6. Corollary 4.5 appears rather implicitly in [TV97, Appendix B], as a special case of a much more general result. More precisely, it is the case $\ell=1$ of an infinite family of evaluations for the determinants

$$
\operatorname{det}_{\mathfrak{l}, \mathfrak{m} \in \mathcal{Z}_{\ell}^{n}}\left(J_{\mathfrak{l}}(u \triangleright \mathfrak{m})\right)
$$

where rows and columns are labelled by the compositions

$$
\mathcal{Z}_{\ell}^{n}=\left\{\mathfrak{l}=\left(\mathfrak{l}_{1}, \ldots, \mathfrak{l}_{n}\right) ; \mathfrak{l}_{i} \geqslant 0, \sum \mathfrak{l}_{i}=\ell\right\} .
$$

When $\ell=1, \mathcal{Z}_{\ell}^{n}$ can be identified with $\{1, \ldots, n\}$ and one gets a 'usual' determinant. For an explanation of the other symbols in (4.3), the reader is kindly referred to [TV97].

If we let $a_{j}=c_{j}$ in Corollary 4.5 and replace $t$ by $t a_{1} \cdots a_{n}$, so that $b_{j}=t a_{j}$, we recover the following determinant evaluation due to Frobenius [Fro82]. This identity has found applications to Ruijsenaars operators [Rui87], to multidimensional elliptic hypergeometric series and integrals [KN03, Rai03] and to number theory [Ros05]. It is closely related to the denominator formula for certain affine superalgebras, see [Ros05]. For a generalization to higher genus Riemann surfaces, see [Fay73, Corollary 2.19].

Corollary 4.7 (An $A$ type Cauchy determinant evaluation [Fro82]). Let $x_{1}, \ldots, x_{n}, a_{1}, \ldots, a_{n}$ and $t$ be indeterminates. Then there holds

$$
\operatorname{det}_{1 \leqslant i, j \leqslant n}\left(\frac{\theta\left(t a_{j} x_{i}\right)}{\theta\left(t, a_{j} x_{i}\right)}\right)=\frac{\theta\left(t a_{1} \cdots a_{n} x_{1} \cdots x_{n}\right)}{\theta(t)} \frac{\prod_{1 \leqslant i<j \leqslant n} a_{j} x_{j} \theta\left(a_{i} / a_{j}, x_{i} / x_{j}\right)}{\prod_{i, j=1}^{n} \theta\left(a_{j} x_{i}\right)} .
$$

Finally, the following result is included here for similar reasons as Corollary 4.3.

Corollary 4.8 (An $A$ type determinant evaluation). Let $x_{1}, \ldots, x_{n}, a_{1}, \ldots, a_{n+1}$ and $b$ be indeterminates. For each $j=1, \ldots, n+1$, let $P_{j}$ be an $A_{j-1}$ theta function of norm $t a_{1} \cdots a_{j}$. Then there holds

$$
\begin{array}{r}
P_{n+1}(b) \underset{1 \leqslant i, j \leqslant n}{\operatorname{det}}\left(P_{j}\left(x_{i}\right) \prod_{k=j+1}^{n+1} \theta\left(a_{k} x_{i}\right)-\frac{P_{n+1}\left(x_{i}\right)}{P_{n+1}(b)} P_{j}(b) \prod_{k=j+1}^{n+1} \theta\left(a_{k} b\right)\right) \\
=\frac{\theta\left(t b a_{1} \cdots a_{n+1} x_{1} \cdots x_{n}\right)}{\theta(t)} \prod_{i=1}^{n+1} P_{i}\left(1 / a_{i}\right) \prod_{1 \leqslant i<j \leqslant n+1} a_{j} x_{j} \theta\left(x_{i} / x_{j}\right),
\end{array}
$$

where $x_{n+1}=b$.

Proof. Proceed as in the proof of Corollary 4.3, but apply Theorem 4.4 instead of Proposition 4.1. 


\section{ElLiptic Determinant EVALUATIONS AND MaCDONALD IDENTITIES}

\subsection{A $C$ type determinant}

The following identity, associated to the affine root system of type $C$, provides a new elliptic extension of the Weyl denominator formulas (1.2b), (1.2c) and (1.2d); see Remark 5.15.

Theorem 4.9 (A $C$ type determinant evaluation). Let $x_{1}, \ldots, x_{n}, a_{1}, \ldots, a_{n}$ and $c_{1}, \ldots, c_{n+2}$ be indeterminates. For each $j=1, \ldots, n$, let $P_{j}$ be an $A_{j-1}$ theta function of norm

$$
\left(c_{1} \cdots c_{n+2} a_{j+1} \cdots a_{n}\right)^{-1} .
$$

Then there holds

$$
\begin{aligned}
& \operatorname{det}_{1 \leqslant i, j \leqslant n}\left(x_{i}^{-n-1} \prod_{k=1}^{n+2} \theta\left(c_{k} x_{i}\right) P_{j}\left(x_{i}\right) \prod_{k=j+1}^{n} \theta\left(a_{k} x_{i}\right)-x_{i}^{n+1} \prod_{k=1}^{n+2} \theta\left(c_{k} x_{i}^{-1}\right) P_{j}\left(x_{i}^{-1}\right) \prod_{k=j+1}^{n} \theta\left(a_{k} x_{i}^{-1}\right)\right) \\
& \quad=\frac{a_{1} \cdots a_{n}}{\theta\left(c_{1} \cdots c_{n+2} a_{1} \cdots a_{n}\right)} \prod_{i=1}^{n} P_{i}\left(1 / a_{i}\right) \prod_{1 \leqslant i<j \leqslant n+2} \theta\left(c_{i} c_{j}\right) \prod_{i=1}^{n} x_{i}^{-1} \theta\left(x_{i}^{2}\right) \prod_{1 \leqslant i<j \leqslant n} a_{j} x_{i}^{-1} \theta\left(x_{i} x_{j}^{ \pm}\right) .
\end{aligned}
$$

Equivalently, factoring $P_{j}$ as in (4.2), we have

$$
\begin{aligned}
& \operatorname{det}_{1 \leqslant i, j \leqslant n}\left(x_{i}^{-n-1} \prod_{k=1}^{n+2} \theta\left(c_{k} x_{i}\right) \prod_{k=1}^{j} \theta\left(b_{k j} x_{i}\right) \prod_{k=j+1}^{n} \theta\left(a_{k} x_{i}\right)-x_{i}^{n+1} \prod_{k=1}^{n+2} \theta\left(c_{k} x_{i}^{-1}\right) \prod_{k=1}^{j} \theta\left(b_{k j} x_{i}^{-1}\right) \prod_{k=j+1}^{n} \theta\left(a_{k} x_{i}^{-1}\right)\right) \\
& =-\frac{1}{c_{1} \cdots c_{n+2}} \prod_{i=2}^{n} \prod_{k=1}^{i} \theta\left(b_{k i} / a_{i}\right) \prod_{1 \leqslant i<j \leqslant n+2} \theta\left(c_{i} c_{j}\right) \prod_{i=1}^{n} x_{i}^{-1} \theta\left(x_{i}^{2}\right) \prod_{1 \leqslant i<j \leqslant n} a_{j} x_{i}^{-1} \theta\left(x_{i} x_{j}^{ \pm}\right),
\end{aligned}
$$

where

$$
b_{1 j} \cdots b_{j j} a_{j+1} \cdots a_{n} c_{1} \cdots c_{n+2}=1, \quad j=1, \ldots, n .
$$

We give two proofs of Theorem 4.9.

First proof of Theorem 4.9. Using Lemma 3.3, one checks that the determinant is of the form (3.5b), with $R=C_{n}$. Proposition 3.4 then guarantees that the quotient of the two sides of (4.5) is a constant, so it is enough to verify the equality for some fixed values of $x_{i}$. We choose $x_{i}=c_{i}$, so that the second term in each matrix element vanishes. The factor $\prod_{k=1}^{n+2} \theta\left(c_{k} x_{i}\right)$ may then be pulled out from the $i$ th row of the determinant and cancelled, using

$$
\prod_{i=1}^{n} \prod_{k=1}^{n+2} \theta\left(c_{k} x_{i}\right)=\frac{1}{\theta\left(c_{n+1} c_{n+2}\right)} \prod_{1 \leqslant i<j \leqslant n+2} \theta\left(c_{i} c_{j}\right) \prod_{i=1}^{n} \theta\left(x_{i}^{2}\right) \prod_{1 \leqslant i<j \leqslant n} \theta\left(x_{i} x_{j}\right) .
$$

Introducing the parameter $t=1 / c_{1} \cdots c_{n+2} a_{1} \cdots a_{n}$, we note that

$$
\frac{\theta\left(c_{n+1} c_{n+2}\right)}{\theta\left(c_{1} \cdots c_{n+2} a_{1} \cdots a_{n}\right)}=\frac{\theta\left(t a_{1} \cdots a_{n} x_{1} \cdots x_{n}\right)}{\theta(t)} \prod_{i=1}^{n} \frac{1}{a_{i} x_{i}} .
$$

Thus, we are reduced to proving

$$
\operatorname{det}_{1 \leqslant i, j \leqslant n}\left(P_{j}\left(x_{i}\right) \prod_{k=j+1}^{n} \theta\left(a_{k} x_{i}\right)\right)=\frac{\theta\left(t a_{1} \cdots a_{n} x_{1} \cdots x_{n}\right)}{\theta(t)} \prod_{i=1}^{n} P_{i}\left(1 / a_{i}\right) \prod_{1 \leqslant i<j \leqslant n} a_{j} x_{j} \theta\left(x_{i} / x_{j}\right),
$$

where $P_{j}$ is an $A_{j-1}$ theta function of norm $t a_{1} \cdots a_{j}$, and where $x_{j}$ may again be viewed as free variables. This is exactly Theorem 4.4 . 


\section{H. Rosengren And M. Schlosser}

Let $\mathcal{R}_{i}$ denote the reflection operator $\mathcal{R}_{i} f\left(x_{i}\right)=f\left(x_{i}^{-1}\right)$. Then, due to linearity of the determinant, the left-hand side of (4.5) may be written

$$
\begin{aligned}
\prod_{i=1}^{n}(1 & \left.-\mathcal{R}_{i}\right) \prod_{i=1}^{n}\left(x_{i}^{-n-1} \prod_{k=1}^{n+2} \theta\left(c_{k} x_{i}\right)\right) \operatorname{det}_{1 \leqslant i, j \leqslant n}\left(P_{j}\left(x_{i}\right) \prod_{k=j+1}^{n} \theta\left(a_{k} x_{i}\right)\right) \\
= & \frac{1}{\theta\left(1 / c_{1} \cdots c_{n+2} a_{1} \cdots a_{n}\right)} \prod_{i=1}^{n} P_{i}\left(1 / a_{i}\right) \prod_{i=1}^{n}\left(1-\mathcal{R}_{i}\right) \theta\left(\frac{x_{1} \cdots x_{n}}{c_{1} \cdots c_{n+2}}\right) \\
& \times \prod_{i=1}^{n}\left(x_{i}^{-n-1} \prod_{k=1}^{n+2} \theta\left(c_{k} x_{i}\right)\right) \prod_{1 \leqslant i<j \leqslant n} a_{j} x_{j} \theta\left(x_{i} / x_{j}\right),
\end{aligned}
$$

where we used Theorem 4.4 to compute the determinant. Comparing this with the right-hand side of (4.5) gives the following equivalent form of Theorem 4.9.

Corollary 4.10. In the notation above,

$$
\begin{aligned}
\prod_{i=1}^{n}(1 & \left.-\mathcal{R}_{i}\right) \theta\left(\frac{x_{1} \cdots x_{n}}{c_{1} \cdots c_{n+2}}\right) \prod_{i=1}^{n}\left(x_{i}^{-n-1} \prod_{j=1}^{n+2} \theta\left(c_{j} x_{i}\right)\right) \prod_{1 \leqslant i<j \leqslant n} x_{j} \theta\left(x_{i} / x_{j}\right) \\
& =-\frac{1}{c_{1} \cdots c_{n+2}} \prod_{1 \leqslant i<j \leqslant n+2} \theta\left(c_{i} c_{j}\right) \prod_{i=1}^{n} x_{i}^{-1} \theta\left(x_{i}^{2}\right) \prod_{1 \leqslant i<j \leqslant n} x_{i}^{-1} \theta\left(x_{i} x_{j}^{ \pm}\right) .
\end{aligned}
$$

Corollary 4.10 resembles some identities in the work of Rains [Rai03]. It can be used to give an alternative proof of his type I $B C_{n}$ integral, originally conjectured by van Diejen and Spiridonov [DS01] (Rains, personal communication). It would be interesting to know whether Corollary 4.10 can be obtained by specializing a multidimensional elliptic hypergeometric summation theorem on $0 \leqslant k_{i} \leqslant m_{i}(i=1, \ldots, n)$ to the case $m_{i} \equiv 1$.

One consequence of (4.6) is that if we can compute the left-hand side for some special choice of $a_{j}$ and $P_{j}$, we can compute it in general, since $a_{j}$ and $P_{j}$ appear trivially on the right-hand side. This observation can be used to give an alternative proof of Theorem 4.9, based on the type $D$ Cauchy determinant of Corollary 4.2 .

Second proof of Theorem 4.9. We consider the special case when $a_{j}=c_{j}^{-1}, 1 \leqslant j \leqslant n$, and

$$
P_{j}(x)=\theta\left(t c_{j}^{-1} x\right) \prod_{k=1}^{j-1} \theta\left(c_{k}^{-1} x\right)
$$

where $t c_{n+1} c_{n+2}=1$. Then, the left-hand side of (4.5) can be written

$$
\operatorname{det}_{1 \leqslant i, j \leqslant n}\left(\left(\prod_{k=1, k \neq j}^{n} c_{k}^{-1} \theta\left(c_{k} x_{i}^{ \pm}\right)\right)\left(1-\mathcal{R}_{i}\right) x_{i}^{-2} \theta\left(c_{n+1} x_{i}, c_{n+2} x_{i}, c_{j} x_{i}, t c_{j}^{-1} x_{i}\right)\right) .
$$

By (3.4) and Corollary 4.2, this equals

$$
\begin{aligned}
\operatorname{det}_{1 \leqslant i, j \leqslant n} & \left(\left(\prod_{k=1, k \neq j}^{n} c_{k}^{-1} \theta\left(c_{k} x_{i}^{ \pm}\right)\right) x_{i}^{-1} c_{j}^{-1} \theta\left(x_{i}^{2}, t, c_{j} c_{n+1}, c_{j} c_{n+2}\right)\right) \\
= & \frac{\theta(t)^{n}}{c_{1}^{n} \cdots c_{n}^{n}} \prod_{i=1}^{n} x_{i}^{-1} \theta\left(x_{i}^{2}, c_{i} c_{n+1}, c_{i} c_{n+2}\right) \prod_{i, j=1}^{n} \theta\left(c_{j} x_{i}^{ \pm}\right) \operatorname{det}_{1 \leqslant i, j \leqslant n}\left(\frac{1}{\theta\left(c_{j} x_{i}^{ \pm}\right)}\right) \\
= & \frac{\theta(t)^{n}}{c_{1}^{n} \cdots c_{n}^{n}} \prod_{i=1}^{n} x_{i}^{-1} \theta\left(x_{i}^{2}, c_{i} c_{n+1}, c_{i} c_{n+2}\right) \prod_{1 \leqslant i<j \leqslant n} c_{j} x_{j}^{-1} \theta\left(x_{j} x_{i}^{ \pm}, c_{i} c_{j}^{ \pm}\right),
\end{aligned}
$$




\section{Elliptic Determinant EVALUATIONS AND MaCDOnAld identities}

which agrees with the right-hand side of (4.5). As was remarked above, the general case now follows using (4.6).

\subsection{Determinants of type $B, B^{\vee}, C^{\vee}, B C$ and $D$}

If $c^{2} \in p^{\mathbb{Z}}$, then $\theta(c x)$ and $\theta(c / x)$ are equal up to a trivial factor. Thus, if one of the parameters $c_{j}$ in Theorem 4.9 is of this form, then the factor $\prod_{i=1}^{n} \theta\left(c_{j} x_{i}\right)$ may be pulled out from the determinant. Up to the trivial scaling $c_{j} \mapsto p c_{j}$, there are four choices: $c_{j} \in\left\{1,-1, p^{\frac{1}{2}},-p^{\frac{1}{2}}\right\}$. By $(2.4), \theta\left(c_{j} x_{i}\right)$ then cancels against a part of the factor $\theta\left(x_{i}^{2}\right)$ on the right-hand side. Making various specializations of this sort, the $C_{n}$ Macdonald denominator in (4.5) can be reduced to the Macdonald denominator for $B_{n}, B_{n}^{\vee}, C_{n}^{\vee}, B C_{n}$ and $D_{n}$.

As a first example, we let $c_{n+2}=-1$ in Theorem 4.9. Then,

$$
\frac{\theta\left(x_{i}^{2}\right)}{\theta\left(c_{n+2} x_{i}\right)}=\theta\left(x_{i}\right) \theta\left(p x_{i}^{2} ; p^{2}\right) .
$$

This gives the following determinant of type $B C$.

Corollary 4.11 (A $B C$ type determinant evaluation). Let $x_{1}, \ldots, x_{n}, a_{1}, \ldots, a_{n}$ and $c_{1}, \ldots, c_{n+1}$ be indeterminates. For each $j=1, \ldots, n$, let $P_{j}$ be an $A_{j-1}$ theta function of norm

$$
-\left(c_{1} \cdots c_{n+1} a_{j+1} \cdots a_{n}\right)^{-1} \text {. }
$$

Then there holds

$$
\begin{aligned}
\operatorname{det}_{1 \leqslant i, j \leqslant n} & \left(x_{i}^{-n} \prod_{k=1}^{n+1} \theta\left(c_{k} x_{i}\right) P_{j}\left(x_{i}\right) \prod_{k=j+1}^{n} \theta\left(a_{k} x_{i}\right)-x_{i}^{n+1} \prod_{k=1}^{n+1} \theta\left(c_{k} x_{i}^{-1}\right) P_{j}\left(x_{i}^{-1}\right) \prod_{k=j+1}^{n} \theta\left(a_{k} x_{i}^{-1}\right)\right) \\
= & \frac{a_{1} \cdots a_{n}}{\theta\left(-c_{1} \cdots c_{n+1} a_{1} \cdots a_{n}\right)} \prod_{i=1}^{n} P_{i}\left(1 / a_{i}\right) \prod_{i=1}^{n+1} \theta\left(-c_{i}\right) \prod_{1 \leqslant i<j \leqslant n+1} \theta\left(c_{i} c_{j}\right) \prod_{i=1}^{n} \theta\left(x_{i}\right) \theta\left(p x_{i}^{2} ; p^{2}\right) \\
& \times \prod_{1 \leqslant i<j \leqslant n} a_{j} x_{i}^{-1} \theta\left(x_{i} x_{j}^{ \pm}\right) .
\end{aligned}
$$

If we let $c_{n+1}=-p^{\frac{1}{2}}$ in Corollary 4.11, we obtain the following determinant of type $C^{\vee}$.

Corollary 4.12 (A $C^{\vee}$ type determinant evaluation). Let $x_{1}, \ldots, x_{n}, a_{1}, \ldots, a_{n}$ and $c_{1}, \ldots, c_{n}$ be indeterminates. For each $j=1, \ldots, n$, let $P_{j}$ be an $A_{j-1}$ theta function of norm

$$
\left(p^{\frac{1}{2}} c_{1} \cdots c_{n} a_{j+1} \cdots a_{n}\right)^{-1} .
$$

Then there holds

$$
\begin{aligned}
\operatorname{det}_{1 \leqslant i, j \leqslant n} & \left(x_{i}^{-n} \prod_{k=1}^{n} \theta\left(c_{k} x_{i}\right) P_{j}\left(x_{i}\right) \prod_{k=j+1}^{n} \theta\left(a_{k} x_{i}\right)-x_{i}^{n+1} \prod_{k=1}^{n} \theta\left(c_{k} x_{i}^{-1}\right) P_{j}\left(x_{i}^{-1}\right) \prod_{k=j+1}^{n} \theta\left(a_{k} x_{i}^{-1}\right)\right) \\
= & \frac{a_{1} \cdots a_{n} \theta\left(p^{\frac{1}{2}}\right)}{\theta\left(p^{\frac{1}{2}} c_{1} \cdots c_{n} a_{1} \cdots a_{n}\right)} \prod_{i=1}^{n} P_{i}\left(1 / a_{i}\right) \prod_{i=1}^{n} \theta\left(-c_{i}, p^{\frac{1}{2}}\right) \prod_{1 \leqslant i<j \leqslant n} \theta\left(c_{i} c_{j}\right) \prod_{i=1}^{n} \theta\left(x_{i} ; p^{\frac{1}{2}}\right) \\
& \times \prod_{1 \leqslant i<j \leqslant n} a_{j} x_{i}^{-1} \theta\left(x_{i} x_{j}^{ \pm}\right) .
\end{aligned}
$$

If we let $c_{n+1}=-p^{\frac{1}{2}}$ and $c_{n+2}=p^{\frac{1}{2}}$ in Theorem 4.9 , and replace $c_{1}$ by $c_{1} / p$ for convenience, we obtain the following determinant of type $B^{\vee}$. 


\section{H. Rosengren And M. Schlosser}

Corollary 4.13 (A $B^{\vee}$ type determinant evaluation). Let $x_{1}, \ldots, x_{n}, a_{1}, \ldots, a_{n}$ and $c_{1}, \ldots, c_{n}$ be indeterminates. For each $j=1, \ldots, n$, let $P_{j}$ be an $A_{j-1}$ theta function of norm

$$
-\left(c_{1} \cdots c_{n} a_{j+1} \cdots a_{n}\right)^{-1} \text {. }
$$

Then there holds

$$
\begin{aligned}
\operatorname{det}_{1 \leqslant i, j \leqslant n} & \left(x_{i}^{-n} \prod_{k=1}^{n} \theta\left(c_{k} x_{i}\right) P_{j}\left(x_{i}\right) \prod_{k=j+1}^{n} \theta\left(a_{k} x_{i}\right)-x_{i}^{n} \prod_{k=1}^{n} \theta\left(c_{k} x_{i}^{-1}\right) P_{j}\left(x_{i}^{-1}\right) \prod_{k=j+1}^{n} \theta\left(a_{k} x_{i}^{-1}\right)\right) \\
= & \frac{a_{1} \cdots a_{n} c_{1} \cdots c_{n} \theta(-1)}{\theta\left(-c_{1} \cdots c_{n} a_{1} \cdots a_{n}\right)} \prod_{i=1}^{n} P_{i}\left(1 / a_{i}\right) \prod_{i=1}^{n} \theta\left(p c_{i}^{2} ; p^{2}\right) \prod_{1 \leqslant i<j \leqslant n} \theta\left(c_{i} c_{j}\right) \prod_{i=1}^{n} x_{i}^{-1} \theta\left(x_{i}^{2} ; p^{2}\right) \\
& \times \prod_{1 \leqslant i<j \leqslant n} a_{j} x_{i}^{-1} \theta\left(x_{i} x_{j}^{ \pm}\right) .
\end{aligned}
$$

If we let $c_{n}=-1$ in Corollary 4.13 we obtain, using also (2.5), the following determinant of type $B$.

Corollary 4.14 (A $B$ type determinant evaluation). Let $x_{1}, \ldots, x_{n}, a_{1}, \ldots, a_{n}$ and $c_{1}, \ldots, c_{n-1}$ be indeterminates. For each $j=1, \ldots, n$, let $P_{j}$ be an $A_{j-1}$ theta function of norm

$$
\left(c_{1} \cdots c_{n-1} a_{j+1} \cdots a_{n}\right)^{-1} \text {. }
$$

Then there holds

$$
\begin{aligned}
\operatorname{det}_{1 \leqslant i, j \leqslant n} & \left(x_{i}^{1-n} \prod_{k=1}^{n-1} \theta\left(c_{k} x_{i}\right) P_{j}\left(x_{i}\right) \prod_{k=j+1}^{n} \theta\left(a_{k} x_{i}\right)-x_{i}^{n} \prod_{k=1}^{n-1} \theta\left(c_{k} x_{i}^{-1}\right) P_{j}\left(x_{i}^{-1}\right) \prod_{k=j+1}^{n} \theta\left(a_{k} x_{i}^{-1}\right)\right) \\
= & -\frac{2 a_{1} \cdots a_{n} c_{1} \cdots c_{n-1}}{\theta\left(c_{1} \cdots c_{n-1} a_{1} \cdots a_{n}\right)} \prod_{i=1}^{n} P_{i}\left(1 / a_{i}\right) \prod_{i=1}^{n-1} \theta\left(-c_{i}\right) \theta\left(p c_{i}^{2} ; p^{2}\right) \prod_{1 \leqslant i<j \leqslant n-1} \theta\left(c_{i} c_{j}\right) \prod_{i=1}^{n} \theta\left(x_{i}\right) \\
& \times \prod_{1 \leqslant i<j \leqslant n} a_{j} x_{i}^{-1} \theta\left(x_{i} x_{j}^{ \pm}\right) .
\end{aligned}
$$

Finally, assuming $n \geqslant 2$, we let $c_{n-1}=1$ in Corollary 4.14. Again using (2.5), we obtain following type $D$ determinant.

Corollary 4.15 (A $D$ type determinant evaluation). Let $x_{1}, \ldots, x_{n}, a_{1}, \ldots, a_{n}$ and $c_{1}, \ldots, c_{n-2}$ be indeterminates. For each $j=1, \ldots, n$, let $P_{j}$ be an $A_{j-1}$ theta function of norm

$$
\left(c_{1} \cdots c_{n-2} a_{j+1} \cdots a_{n}\right)^{-1} \text {. }
$$

Then, for $n \geqslant 2$, there holds

$$
\begin{aligned}
\operatorname{det}_{1 \leqslant i, j \leqslant n} & \left(x_{i}^{1-n} \prod_{k=1}^{n-2} \theta\left(c_{k} x_{i}\right) P_{j}\left(x_{i}\right) \prod_{k=j+1}^{n} \theta\left(a_{k} x_{i}\right)+x_{i}^{n-1} \prod_{k=1}^{n-2} \theta\left(c_{k} x_{i}^{-1}\right) P_{j}\left(x_{i}^{-1}\right) \prod_{k=j+1}^{n} \theta\left(a_{k} x_{i}^{-1}\right)\right) \\
& =-\frac{4 a_{1} \cdots a_{n} c_{1} \cdots c_{n-2}}{\theta\left(c_{1} \cdots c_{n-2} a_{1} \cdots a_{n}\right)} \prod_{i=1}^{n} P_{i}\left(1 / a_{i}\right) \prod_{1 \leqslant i \leqslant j \leqslant n-2} \theta\left(c_{i} c_{j}\right) \prod_{1 \leqslant i<j \leqslant n} a_{j} x_{i}^{-1} \theta\left(x_{i} x_{j}^{ \pm}\right) .
\end{aligned}
$$

\section{Some polynomial determinant evaluations}

In this section we consider the polynomial special case, $p=0$, of the elliptic determinant evaluations in $\S 4$. The resulting identities involve the Weyl denominator of classical (non-affine) root systems, cf. (1.2). 


\section{Elliptic DETERMinANT EVALUATIONS AND MACDONALD IDENTITIES}

We must first interpret the term ' $A_{n-1}$ theta function' in the case $p=0$. One way is to rewrite Definition 3.1 in terms of the Laurent coefficients of $f(x)=\sum_{j} a_{j} x^{j}$. Namely, $f$ is an $A_{n-1}$ theta function of norm $t$ if and only if

$$
a_{j+n}=(-1)^{n} t p^{j} a_{j}
$$

When $p=0$ this means that $a_{j}=0$ unless $0 \leqslant j \leqslant n$ and that $a_{n}=(-1)^{n} t a_{0}$. Thus, we obtain precisely the space of polynomials of degree $n$ and norm $t$, where the norm of $a_{0}+a_{1} x+\cdots+a_{n} x^{n}$ is defined as $(-1)^{n} a_{n} / a_{0}$. Equivalently, the polynomial $C\left(1-b_{1} x\right) \cdots\left(1-b_{n} x\right)$ has norm $b_{1} \cdots b_{n}$. Thus, we obtain the same result by formally letting $p=0$ in Lemma 3.2. With this interpretation of the term $A_{n-1}$ theta function, Theorems 4.4 and 4.9 remain valid when $p=0$.

\subsection{Determinants of type $A$}

We first give the case $p=0$ of Theorem 4.4.

Corollary 5.1 (An $A$ type determinant evaluation). Let $x_{1}, \ldots, x_{n}, a_{1}, \ldots, a_{n}$ and $t$ be indeterminates. For each $j=1, \ldots, n$, let $P_{j}$ be a polynomial of degree $j$ and norm $t a_{1} \cdots a_{j}$. Then there holds

$$
\operatorname{det}_{1 \leqslant i, j \leqslant n}\left(P_{j}\left(x_{i}\right) \prod_{k=j+1}^{n}\left(1-a_{k} x_{i}\right)\right)=\frac{1-t a_{1} \cdots a_{n} x_{1} \cdots x_{n}}{1-t} \prod_{i=1}^{n} P_{i}\left(1 / a_{i}\right) \prod_{1 \leqslant i<j \leqslant n} a_{j}\left(x_{j}-x_{i}\right) .
$$

It is easy to prove Corollary 5.1 directly by a standard 'identification of factors' argument.

It is possible to remove the restriction on the norm of the polynomials $P_{j}$ through a limit transition, decreasing their degree by one. Such limits do not make sense in the elliptic case $(p \neq 0)$. This leads to the following determinant evaluation due to Krattenthaler [Kra95, Lemma 35], who obtained it as a limit case of [Kra95, Lemma 34], see the discussion of Proposition 4.1 above.

Corollary 5.2 (An $A$ type determinant evaluation $\left[\right.$ Kra95]). Let $x_{1}, \ldots, x_{n}$ and $a_{1}, \ldots, a_{n}$ be indeterminates. For each $j=1, \ldots, n$, let $P_{j-1}$ be a polynomial of degree at most $j-1$. Then there holds

$$
\operatorname{det}_{1 \leqslant i, j \leqslant n}\left(P_{j-1}\left(x_{i}\right) \prod_{k=j+1}^{n}\left(1-a_{k} x_{i}\right)\right)=\prod_{i=1}^{n} P_{i-1}\left(1 / a_{i}\right) \prod_{1 \leqslant i<j \leqslant n} a_{j}\left(x_{j}-x_{i}\right) .
$$

Proof. In Corollary 5.1, write $P_{j}(x)=\left(1-t b_{j} x\right) \tilde{P}_{j-1}(x)$, let $t \rightarrow 0$ and then relabel $\tilde{P}_{j-1} \mapsto P_{j-1}$.

We also note the following consequence of Corollary 4.8 .

Corollary 5.3 (An $A$ type determinant evaluation). Let $x_{1}, \ldots, x_{n}$ and $b$ be indeterminates. For each $j=1, \ldots, n$, let $P_{j-1}(x)$ be a polynomial in $x$ of degree at most $j-1$ with constant term 1 , and let $Q(x)=\left(1-y_{1} x\right) \cdots\left(1-y_{n+1} x\right)$. Then there holds

$$
\begin{aligned}
Q(b) & \operatorname{det}_{1 \leqslant i, j \leqslant n}\left(x_{i}^{n+1-j} P_{j-1}\left(x_{i}\right)-b^{n+1-j} P_{j-1}(b) \frac{Q\left(x_{i}\right)}{Q(b)}\right) \\
& =\left(1-b x_{1} \cdots x_{n} y_{1} \cdots y_{n+1}\right) \prod_{i=1}^{n}\left(x_{i}-b\right) \prod_{1 \leqslant i<j \leqslant n}\left(x_{i}-x_{j}\right) .
\end{aligned}
$$

Proof. In Corollary 4.8, let $p=0$ and assume, as a matter of normalization, that the polynomials $P_{j}$ have constant term 1 . Write $t=s^{n+1}, a_{i}=c_{i} / s$,

$$
\begin{gathered}
P_{j}(x)=\left(1-s^{n+1-j} d_{j} x\right) \tilde{P}_{j-1}(x), \quad j=1, \ldots, n, \\
P_{n+1}(x)=\left(1-y_{1} x\right) \cdots\left(1-y_{n+1} x\right) .
\end{gathered}
$$




\section{H. Rosengren And M. Schlosser}

Then, $\tilde{P}_{j-1}$ has norm $c_{1} \cdots c_{j} / d_{j}$ and $P_{n+1}$ norm $y_{1} \cdots y_{n+1}=c_{1} \cdots c_{n+1}$, which are in particular independent of $s$. Dividing both sides of (4.4) by $\prod_{1 \leqslant i<j \leqslant n+1}\left(-a_{j}\right)$, letting $s \rightarrow 0$ and finally relabelling $\tilde{P}_{j-1} \mapsto P_{j-1}, P_{n+1} \mapsto Q$, we obtain the desired result.

Remark 5.4. Note that the right-hand side of (5.1) is independent of $P_{j-1}$. The special case $P_{j-1}(x)=1$, for $j=1, \ldots, n$, is Lemma A.1 of [Sch97], which was needed in order to obtain an $A_{n}$ matrix inversion that played a crucial role in the derivation of multiple basic hypergeometric series identities. A slight generalization of [Sch97, Lemma A.1] was given in [Sch00a, Lemma A.1].

\subsection{Determinants of type $B, C$ and $D$}

Next, we turn to the $p=0$ case of Theorem 4.9.

Corollary 5.5 (A $C$ type determinant evaluation). Let $x_{1}, \ldots, x_{n}, a_{1}, \ldots, a_{n}$ and $c_{1}, \ldots, c_{n+2}$ be indeterminates. For each $j=1, \ldots, n$, let $P_{j}$ be a polynomial of degree $j$ with norm

$$
\left(c_{1} \cdots c_{n+2} a_{j+1} \cdots a_{n}\right)^{-1} \text {. }
$$

Then there holds

$$
\begin{aligned}
\operatorname{det}_{1 \leqslant i, j \leqslant n} & \left(x_{i}^{-n-1} \prod_{k=1}^{n+2}\left(1-c_{k} x_{i}\right) P_{j}\left(x_{i}\right) \prod_{k=j+1}^{n}\left(1-a_{k} x_{i}\right)-x_{i}^{n+1} \prod_{k=1}^{n+2}\left(1-c_{k} x_{i}^{-1}\right) P_{j}\left(x_{i}^{-1}\right) \prod_{k=j+1}^{n}\left(1-a_{k} x_{i}^{-1}\right)\right) \\
= & \frac{a_{1} \cdots a_{n}}{1-c_{1} \cdots c_{n+2} a_{1} \cdots a_{n}} \prod_{i=1}^{n} P_{i}\left(1 / a_{i}\right) \prod_{1 \leqslant i<j \leqslant n+2}\left(1-c_{i} c_{j}\right) \prod_{i=1}^{n} x_{i}^{-n}\left(1-x_{i}^{2}\right) \\
& \times \prod_{1 \leqslant i<j \leqslant n} a_{j}\left(x_{j}-x_{i}\right)\left(1-x_{i} x_{j}\right) .
\end{aligned}
$$

If we let $c_{n+2}=-1$ in Corollary 5.5 or, equivalently, $p=0$ in Corollary 4.11 , we obtain the following determinant of type $B$.

Corollary 5.6 (A $B$ type determinant evaluation). Let $x_{1}, \ldots, x_{n}, a_{1}, \ldots, a_{n}$ and $c_{1}, \ldots, c_{n+1}$ be indeterminates. For each $j=1, \ldots, n$, let $P_{j}$ be a polynomial of degree $j$ with norm

$$
-\left(c_{1} \cdots c_{n+1} a_{j+1} \cdots a_{n}\right)^{-1} \text {. }
$$

Then there holds

$$
\begin{aligned}
\operatorname{det}_{1 \leqslant i, j \leqslant n} & \left(x_{i}^{-n} \prod_{k=1}^{n+1}\left(1-c_{k} x_{i}\right) P_{j}\left(x_{i}\right) \prod_{k=j+1}^{n}\left(1-a_{k} x_{i}\right)-x_{i}^{n+1} \prod_{k=1}^{n+1}\left(1-c_{k} x_{i}^{-1}\right) P_{j}\left(x_{i}^{-1}\right) \prod_{k=j+1}^{n}\left(1-a_{k} x_{i}^{-1}\right)\right) \\
= & \frac{a_{1} \cdots a_{n}}{1+c_{1} \cdots c_{n+1} a_{1} \cdots a_{n}} \prod_{i=1}^{n} P_{i}\left(1 / a_{i}\right) \prod_{1 \leqslant i<j \leqslant n+1}\left(1-c_{i} c_{j}\right) \prod_{i=1}^{n+1}\left(1+c_{i}\right) \prod_{i=1}^{n} x_{i}^{1-n}\left(1-x_{i}\right) \\
& \times \prod_{1 \leqslant i<j \leqslant n} a_{j}\left(x_{j}-x_{i}\right)\left(1-x_{i} x_{j}\right) .
\end{aligned}
$$

If we let $c_{n+1}=1$ in Corollary 5.6, the factor $\prod_{i=1}^{n}\left(1-x_{i}\right)$ may be cancelled. This gives the following determinant of type $D$.

Corollary 5.7 (A $D$ type determinant evaluation). Let $x_{1}, \ldots, x_{n}, a_{1}, \ldots, a_{n}$ and $c_{1}, \ldots, c_{n}$ be indeterminates. For each $j=1, \ldots, n$, let $P_{j}$ be a polynomial of degree $j$ with norm

$$
-\left(c_{1} \cdots c_{n} a_{j+1} \cdots a_{n}\right)^{-1} \text {. }
$$




\section{Elliptic DETERMinANT EVALUATIONS AND MACDONALD IDENTITIES}

Then there holds

$$
\begin{aligned}
\underset{1 \leqslant i, j \leqslant n}{\operatorname{det}}\left(x_{i}^{-n} \prod_{k=1}^{n}\left(1-c_{k} x_{i}\right) P_{j}\left(x_{i}\right) \prod_{k=j+1}^{n}\left(1-a_{k} x_{i}\right)+x_{i}^{n} \prod_{k=1}^{n}\left(1-c_{k} x_{i}^{-1}\right) P_{j}\left(x_{i}^{-1}\right) \prod_{k=j+1}^{n}\left(1-a_{k} x_{i}^{-1}\right)\right) \\
\quad=\frac{2 a_{1} \cdots a_{n}}{1+c_{1} \cdots c_{n} a_{1} \cdots a_{n}} \prod_{i=1}^{n} P_{i}\left(1 / a_{i}\right) \prod_{1 \leqslant i \leqslant j \leqslant n}\left(1-c_{i} c_{j}\right) \prod_{i=1}^{n} x_{i}^{1-n} \prod_{1 \leqslant i<j \leqslant n} a_{j}\left(x_{j}-x_{i}\right)\left(1-x_{i} x_{j}\right) .
\end{aligned}
$$

Similarly as when deriving Corollary 5.2 from Corollary 5.1, we may remove the restriction on the norm of $P_{j}$ in Corollaries 5.5, 5.6 and 5.7 by a limit transition, through which their degree is lowered by one.

Corollary 5.8 (A $C$ type determinant evaluation). Let $x_{1}, \ldots, x_{n}, a_{1}, \ldots, a_{n}$ and $c_{1}, \ldots, c_{n+1}$ be indeterminates. For each $j=1, \ldots, n$, let $P_{j-1}$ be a polynomial of degree at most $j-1$. Then there holds

$$
\begin{aligned}
\operatorname{det}_{1 \leqslant i, j \leqslant n} & \left(x_{i}^{-n} \prod_{k=1}^{n+1}\left(1-c_{k} x_{i}\right) P_{j-1}\left(x_{i}\right) \prod_{k=j+1}^{n}\left(1-a_{k} x_{i}\right)-x_{i}^{n} \prod_{k=1}^{n+1}\left(1-c_{k} x_{i}^{-1}\right) P_{j-1}\left(x_{i}^{-1}\right) \prod_{k=j+1}^{n}\left(1-a_{k} x_{i}^{-1}\right)\right) \\
& =\prod_{i=1}^{n} P_{i-1}\left(1 / a_{i}\right) \prod_{1 \leqslant i<j \leqslant n+1}\left(1-c_{i} c_{j}\right) \prod_{i=1}^{n} x_{i}^{-n}\left(1-x_{i}^{2}\right) \prod_{1 \leqslant i<j \leqslant n} a_{j}\left(x_{j}-x_{i}\right)\left(1-x_{i} x_{j}\right) .
\end{aligned}
$$

Proof. In Corollary 5.5, write $P_{j}(x)=\left(x+b_{j} c_{n+2}\right) \tilde{P}_{j-1}(x)$, let $c_{n+2} \rightarrow 0$ and relabel $\tilde{P}_{j-1} \mapsto$ $P_{j-1}$.

Corollary 5.9 (A $B$ type determinant evaluation). Let $x_{1}, \ldots, x_{n}, a_{1}, \ldots, a_{n}$ and $c_{1}, \ldots, c_{n}$ be indeterminates. For each $j=1, \ldots, n$, let $P_{j-1}$ be a polynomial of degree at most $j-1$. Then there holds

$$
\begin{aligned}
\operatorname{det}_{1 \leqslant i, j \leqslant n} & \left(x_{i}^{1-n} \prod_{k=1}^{n}\left(1-c_{k} x_{i}\right) P_{j-1}\left(x_{i}\right) \prod_{k=j+1}^{n}\left(1-a_{k} x_{i}\right)-x_{i}^{n} \prod_{k=1}^{n}\left(1-c_{k} x_{i}^{-1}\right) P_{j-1}\left(x_{i}^{-1}\right) \prod_{k=j+1}^{n}\left(1-a_{k} x_{i}^{-1}\right)\right) \\
= & \prod_{i=1}^{n} P_{i-1}\left(1 / a_{i}\right) \prod_{1 \leqslant i<j \leqslant n}\left(1-c_{i} c_{j}\right) \prod_{i=1}^{n}\left(1+c_{i}\right) \prod_{i=1}^{n} x_{i}^{1-n}\left(1-x_{i}\right) \prod_{1 \leqslant i<j \leqslant n} a_{j}\left(x_{j}-x_{i}\right)\left(1-x_{i} x_{j}\right) .
\end{aligned}
$$

Proof. Let $c_{n+1}=-1$ in Corollary 5.8 and divide by $\prod_{i=1}^{n}\left(1+x_{i}^{-1}\right)$.

Corollary 5.10 (A $D$ type determinant evaluation). Let $x_{1}, \ldots, x_{n}, a_{1}, \ldots, a_{n}$ and $c_{1}, \ldots, c_{n-1}$ be indeterminates. For each $j=1, \ldots, n$, let $P_{j-1}$ be a polynomial of degree at most $j-1$. Then there holds

$$
\begin{aligned}
\operatorname{det}_{1 \leqslant i, j \leqslant n} & \left(x_{i}^{1-n} \prod_{k=1}^{n-1}\left(1-c_{k} x_{i}\right) P_{j-1}\left(x_{i}\right) \prod_{k=j+1}^{n}\left(1-a_{k} x_{i}\right)\right. \\
& \left.+x_{i}^{n-1} \prod_{k=1}^{n-1}\left(1-c_{k} x_{i}^{-1}\right) P_{j-1}\left(x_{i}^{-1}\right) \prod_{k=j+1}^{n}\left(1-a_{k} x_{i}^{-1}\right)\right) \\
= & 2 \prod_{i=1}^{n} P_{i-1}\left(1 / a_{i}\right) \prod_{1 \leqslant i \leqslant j \leqslant n-1}\left(1-c_{i} c_{j}\right) \prod_{i=1}^{n} x_{i}^{1-n} \prod_{1 \leqslant i<j \leqslant n} a_{j}\left(x_{j}-x_{i}\right)\left(1-x_{i} x_{j}\right) .
\end{aligned}
$$

Proof. Let $c_{n}=1$ in Corollary 5.9 and divide by $\prod_{i=1}^{n}\left(1-x_{i}\right)$.

Next, we give some further specializations of our determinant evaluations, which are closer to the classical Weyl denominator formulas. 


\section{H. Rosengren And M. Schlosser}

Corollary 5.11 (A $C$ type determinant evaluation). Let $x_{1}, \ldots, x_{n}$ and $c_{1}, \ldots, c_{n+1}$ be indeterminates. For each $j=1, \ldots, n$, let $P_{j-1}$ be a polynomial of degree at most $j-1$. Then there holds

$$
\begin{aligned}
\operatorname{det}_{1 \leqslant i, j \leqslant n} & \left(x_{i}^{-j} \prod_{k=1}^{n+1}\left(1-c_{k} x_{i}\right) P_{j-1}\left(x_{i}\right)-x_{i}^{j} \prod_{k=1}^{n+1}\left(1-c_{k} x_{i}^{-1}\right) P_{j-1}\left(x_{i}^{-1}\right)\right) \\
& =\prod_{i=1}^{n} P_{i-1}(0) \prod_{1 \leqslant i<j \leqslant n+1}\left(1-c_{i} c_{j}\right) \prod_{i=1}^{n} x_{i}^{-n}\left(1-x_{i}^{2}\right) \prod_{1 \leqslant i<j \leqslant n}\left(x_{i}-x_{j}\right)\left(1-x_{i} x_{j}\right) .
\end{aligned}
$$

Proof. In Corollary 5.8, divide both sides of the identity by $\prod_{1 \leqslant i<j \leqslant n}\left(-a_{j}\right)$, and then let $a_{j} \rightarrow \infty$, successively for $j=2, \ldots, n$.

Remark 5.12. The special case $P_{j-1}(x)=1$, for $j=1, \ldots, n$, is Lemma A.11 of [Sch97], needed in order to obtain a $C_{n}$ matrix inversion (which was later applied in [Sch99]).

Corollary 5.13 (A $B$ type determinant evaluation). Let $x_{1}, \ldots, x_{n}$ and $c_{1}, \ldots, c_{n}$ be indeterminates. For each $j=1, \ldots, n$, let $P_{j-1}$ be a polynomial of degree at most $j-1$. Then there holds

$$
\begin{aligned}
\underset{1 \leqslant i, j \leqslant n}{\operatorname{det}} & \left(x_{i}^{1-j} \prod_{k=1}^{n}\left(1-c_{k} x_{i}\right) P_{j-1}\left(x_{i}\right)-x_{i}^{j} \prod_{k=1}^{n}\left(1-c_{k} x_{i}^{-1}\right) P_{j-1}\left(x_{i}^{-1}\right)\right) \\
= & \prod_{i=1}^{n} P_{i-1}(0) \prod_{1 \leqslant i<j \leqslant n}\left(1-c_{i} c_{j}\right) \prod_{i=1}^{n}\left(1+c_{i}\right) \prod_{i=1}^{n} x_{i}^{1-n}\left(1-x_{i}\right) \prod_{1 \leqslant i<j \leqslant n}\left(x_{i}-x_{j}\right)\left(1-x_{i} x_{j}\right) .
\end{aligned}
$$

Proof. Let $c_{n+1}=-1$ in Corollary 5.11 and divide by $\prod_{i=1}^{n}\left(1+x_{i}^{-1}\right)$.

Corollary 5.14 (A $D$ type determinant evaluation). Let $x_{1}, \ldots, x_{n}$ and $c_{1}, \ldots, c_{n-1}$ be indeterminates. For each $j=1, \ldots, n$, let $P_{j-1}$ be a polynomial of degree at most $j-1$. Then there holds

$$
\begin{aligned}
\operatorname{det}_{1 \leqslant i, j \leqslant n} & \left(x_{i}^{1-j} \prod_{k=1}^{n-1}\left(1-c_{k} x_{i}\right) P_{j-1}\left(x_{i}\right)+x_{i}^{j-1} \prod_{k=1}^{n-1}\left(1-c_{k} x_{i}^{-1}\right) P_{j-1}\left(x_{i}^{-1}\right)\right) \\
= & 2 \prod_{i=1}^{n} P_{i-1}(0) \prod_{1 \leqslant i \leqslant j \leqslant n-1}\left(1-c_{i} c_{j}\right) \prod_{i=1}^{n} x_{i}^{1-n} \prod_{1 \leqslant i<j \leqslant n}\left(x_{i}-x_{j}\right)\left(1-x_{i} x_{j}\right) .
\end{aligned}
$$

Proof. Let $c_{n}=1$ in Corollary 5.13 and divide by $\prod_{i=1}^{n}\left(1-x_{i}\right)$.

Remark 5.15. If we let $c_{j}=0$ and $P_{j}(x)=1$ for all $j$, Corollaries 5.11, 5.13 and 5.14 reduce, up to reversing the order of the columns, to the classical Weyl denominator formulas (1.2c), $(1.2 \mathrm{~b})$ and $(1.2 \mathrm{~d})$, respectively. Similarly, Corollary 5.1 contains (1.2a) as a limit case. Thus, Theorems 4.4 and 4.9 give elliptic extensions of the Weyl denominator formulas for the classical root systems.

\section{The Macdonald identities}

In $\S 4$, we have focused on the left-hand sides of (3.5), trying to find as general families of $R$ theta functions as possible, such that the constant $C$ can be determined. We now focus on the right-hand sides, trying to find a particularly simple expression for $W_{R}$ as a determinant. More precisely, we want the functions $f_{j}$ to have known explicit Laurent expansions, so that the multiple Laurent expansion of $W_{R}$ can be read off from (3.5).

Starting with the case of type $A$, we observe that the function

$$
f_{m}(x)=x^{m} \theta\left((-1)^{n-1} t p^{m} x^{n} ; p^{n}\right),
$$




\section{ElLiptic Determinant EVALUATIONS AND MaCDONALD IDENTITIES}

with $m$ an integer, is an $A_{n-1}$ theta function of norm $t$. Moreover, its Laurent expansion is known from (2.2). Thus, we are led to consider determinants of the form $\operatorname{det}_{i j}\left(f_{m_{j}}\left(x_{i}\right)\right)$, with $m_{j}$ integers, hoping that the constant

$$
C=\frac{\operatorname{det}_{1 \leqslant i, j \leqslant n}\left(f_{m_{j}}\left(x_{i}\right)\right)}{\theta\left(t x_{1} \cdots x_{n}\right) W_{A_{n-1}}(x)}
$$

can be evaluated.

To compute this constant, we specialize the $x_{i}$ to $n$th roots of unity, since the theta functions may then be pulled out from the determinant. To avoid zeroes in the denominator, the $x_{i}$ should be distinct, so we assume that $x_{i}=\omega^{i-1}$, with $\omega$ a primitive $n$th root of unity. By the Vandermonde determinant $(1.2 \mathrm{a})$, we then have

$$
\operatorname{det}_{1 \leqslant i, j \leqslant n}\left(f_{m_{j}}\left(\omega^{i-1}\right)\right)=\prod_{j=1}^{n} \theta\left((-1)^{n-1} t p^{m_{j}} ; p^{n}\right) \prod_{1 \leqslant i<j \leqslant n}\left(\omega^{m_{j}}-\omega^{m_{i}}\right) .
$$

To obtain a non-trivial result, this should be non-zero, so the $m_{i}$ should be equidistributed modulo $n$. Thus, we assume that $m_{i}=i-1$. In that case, by $(2.3)$,

$$
\prod_{j=1}^{n} \theta\left((-1)^{n-1} t p^{m_{j}} ; p^{n}\right)=\theta\left((-1)^{n-1} t\right)=\left.\theta\left(t x_{1} \cdots x_{n}\right)\right|_{x_{i}=\omega^{i-1}},
$$

which gives

$$
\operatorname{det}_{1 \leqslant i, j \leqslant n}\left(x_{i}^{j-1} \theta\left((-1)^{n-1} t p^{j-1} x_{i}^{n} ; p^{n}\right)\right)=\prod_{1 \leqslant i<j \leqslant n} \frac{\omega^{j-1}-\omega^{i-1}}{\omega^{j-1} \theta\left(\omega^{i-j}\right)} W_{A_{n-1}}(x) .
$$

By (2.1), the constant simplifies as

$$
\begin{aligned}
\prod_{1 \leqslant i<j \leqslant n} \frac{\omega^{j-1}-\omega^{i-1}}{\omega^{j-1} \theta\left(\omega^{i-j}\right)} & =\prod_{1 \leqslant i<j \leqslant n} \frac{1}{\left(p \omega^{j-i}, p \omega^{i-j}\right)_{\infty}} \\
& =(p)_{\infty}^{n} \prod_{i, j=1}^{n} \frac{1}{\left(p \omega^{j-i}\right)_{\infty}}=(p)_{\infty}^{n} \prod_{k=1}^{n} \frac{1}{\left(p \omega^{k}\right)_{\infty}^{n}}=\frac{(p ; p)_{\infty}^{n}}{\left(p^{n} ; p^{n}\right)_{\infty}^{n}} .
\end{aligned}
$$

Thus, we arrive at the $A_{n-1}$ case of Proposition 6.1 below.

For the remaining root systems, we consider the case of Proposition 3.4 when the theta functions are constructed using Lemma 3.3, with the corresponding functions $g$ of the form (6.1). By similar arguments as for $A_{n-1}$, one is led to the following determinants, one for each root system.

Proposition 6.1. The following determinant evaluations hold:

$$
\begin{gathered}
\operatorname{det}_{1 \leqslant i, j \leqslant n}\left(x_{i}^{j-1} \theta\left((-1)^{n-1} p^{j-1} t x_{i}^{n} ; p^{n}\right)\right)=\frac{(p ; p)_{\infty}^{n}}{\left(p^{n} ; p^{n}\right)_{\infty}^{n}} \theta\left(t x_{1} \cdots x_{n}\right) W_{A_{n-1}}(x), \\
\operatorname{det}_{1 \leqslant i, j \leqslant n}\left(x_{i}^{j-n} \theta\left(p^{j-1} x_{i}^{2 n-1} ; p^{2 n-1}\right)-x_{i}^{n+1-j} \theta\left(p^{j-1} x_{i}^{1-2 n} ; p^{2 n-1}\right)\right)=\frac{2(p ; p)_{\infty}^{n}}{\left(p^{2 n-1} ; p^{2 n-1}\right)_{\infty}^{n}} W_{B_{n}}(x), \\
\operatorname{det}_{1 \leqslant i, j \leqslant n}\left(x_{i}^{j-n-1} \theta\left(p^{j-1} x_{i}^{2 n} ; p^{2 n}\right)-x_{i}^{n+1-j} \theta\left(p^{j-1} x_{i}^{-2 n} ; p^{2 n}\right)\right)=\frac{2\left(p^{2} ; p^{2}\right)_{\infty}(p ; p)_{\infty}^{n-1}}{\left(p^{2 n} ; p^{2 n}\right)_{\infty}^{n}} W_{B_{n}^{\vee}}(x), \\
\operatorname{det}_{1 \leqslant i, j \leqslant n}\left(x_{i}^{j-n-1} \theta\left(-p^{j} x_{i}^{2 n+2} ; p^{2 n+2}\right)-x_{i}^{n+1-j} \theta\left(-p^{j} x_{i}^{-2 n-2} ; p^{2 n+2}\right)\right)=\frac{(p ; p)_{\infty}^{n}}{\left(p^{2 n+2} ; p^{2 n+2}\right)_{\infty}^{n}} W_{C_{n}}(x), \\
\operatorname{det}_{1 \leqslant i, j \leqslant n}\left(x_{i}^{j-n} \theta\left(-p^{j-\frac{1}{2}} x_{i}^{2 n} ; p^{2 n}\right)-x_{i}^{n+1-j} \theta\left(-p^{j-\frac{1}{2}} x_{i}^{-2 n} ; p^{2 n}\right)\right)=\frac{\left(p^{\frac{1}{2}} ; p^{\frac{1}{2}}\right)_{\infty}(p ; p)_{\infty}^{n-1}}{\left(p^{2 n} ; p^{2 n}\right)_{\infty}^{n}} W_{C_{n}^{\vee}}(x), \\
\operatorname{det}_{1 \leqslant i, j \leqslant n}\left(x_{i}^{j-n} \theta\left(-p^{j} x_{i}^{2 n+1} ; p^{2 n+1}\right)-x_{i}^{n+1-j} \theta\left(-p^{j} x_{i}^{-2 n-1} ; p^{2 n+1}\right)\right)=\frac{(p ; p)_{\infty}^{n}}{\left(p^{2 n+1} ; p^{2 n+1}\right)_{\infty}^{n}} W_{B C_{n}}(x)
\end{gathered}
$$




\section{H. Rosengren And M. Schlosser}

and, for $n \geqslant 2$,

$$
\operatorname{det}_{1 \leqslant i, j \leqslant n}\left(x_{i}^{j-n} \theta\left(-p^{j-1} x_{i}^{2 n-2} ; p^{2 n-2}\right)+x_{i}^{n-j} \theta\left(-p^{j-1} x_{i}^{2-2 n} ; p^{2 n-2}\right)\right)=\frac{4(p ; p)_{\infty}^{n}}{\left(p^{2 n-2} ; p^{2 n-2}\right)_{\infty}^{n}} W_{D_{n}}(x) .
$$

To complete the proof of Proposition 6.1, all that remains is to verify the identities for some fixed values of $x_{i}$. We have already done this for $A_{n-1}$. In general, we proceed exactly as in [Sta89]. Namely, letting $\omega_{k}$ denote a primitive $k$ th root of unity, we specialize $x_{i}$ as $x_{i}=\omega_{4 n-2}^{2 i-1}$ for $R=B_{n}$, $x_{i}=\omega_{4 n}^{2 i-1}$ for $R=B_{n}^{\vee}, x_{i}=\omega_{2 n+2}^{i}$ for $R=C_{n}, x_{i}=\omega_{2 n}^{i}$ for $R=C_{n}^{\vee}, x_{i}=\omega_{2 n+1}^{i}$ for $R=B C_{n}$ and $x_{i}=\omega_{2 n-2}^{i-1}$ for $R=D_{n}$. Under these specializations, the theta functions can be pulled out from the determinants, which are then computed by the Weyl denominator formulas $\left(1.2 \mathrm{~b}\right.$ ) (for $B_{n}, C_{n}^{\vee}$ and $\left.B C_{n}\right),(1.2 \mathrm{c})$ (for $B_{n}^{\vee}$ and $C_{n}$ ) and $\left(1.2 \mathrm{~d}\right.$ ) (for $D_{n}$ ). If we let $Q_{R}$ denote the quotient of the determinant and the expression $W_{R}$, this gives

$$
\begin{aligned}
Q_{B_{n}} & =\frac{\prod_{j=1}^{n} \theta\left(-p^{j-1} ; p^{2 n-1}\right)}{\prod_{j=1}^{n}\left(-p \omega_{2 n-1}^{ \pm(j-n)}\right)_{\infty} \prod_{1 \leqslant i<j \leqslant n}\left(p \omega_{2 n-1}^{j-i}, p \omega_{2 n-1}^{i-j}, p \omega_{2 n-1}^{i+j-1}, p \omega_{2 n-1}^{1-i-j}\right)_{\infty}}, \\
Q_{B_{n}} & =\frac{\prod_{j=1}^{n} \theta\left(-p^{j-1} ; p^{2 n}\right)}{\prod_{j=1}^{n}\left(p^{2} \omega_{2 n}^{ \pm(2 j-1)} ; p^{2}\right)_{\infty} \prod_{1 \leqslant i<j \leqslant n}\left(p \omega_{2 n}^{j-i}, p \omega_{2 n}^{i-j}, p \omega_{2 n}^{i+j-1}, p \omega_{2 n}^{1-i-j}\right)_{\infty}}, \\
Q_{C_{n}} & =\frac{\prod_{j=1}^{n} \theta\left(-p^{j} ; p^{2 n+2}\right)}{\prod_{j=1}^{n}\left(p \omega_{2 n+2}^{ \pm 2 j}\right)_{\infty} \prod_{1 \leqslant i<j \leqslant n}\left(p \omega_{2 n+2}^{j-i}, p \omega_{2 n+2}^{i-j}, p \omega_{2 n+2}^{i+j}, p \omega_{2 n+2}^{-i-j}\right)_{\infty}}, \\
Q_{C_{n}^{\vee}} & =\frac{\prod_{j=1}^{n} \theta\left(-p^{j-\frac{1}{2}} ; p^{2 n}\right)}{\prod_{j=1}^{n}\left(p^{\frac{1}{2}} \omega_{2 n}^{ \pm j} ; p^{\frac{1}{2}}\right)_{\infty} \prod_{1 \leqslant i<j \leqslant n}\left(p \omega_{2 n}^{j-i}, p \omega_{2 n}^{i-j}, p \omega_{2 n}^{i+j}, p \omega_{2 n}^{-i-j}\right)_{\infty}}, \\
Q_{B C_{n}} & =\frac{\prod_{j=1}^{n} \theta\left(-p^{j} ; p^{2 n+1}\right)}{\prod_{j=1}^{n}\left(p \omega_{2 n+1}^{ \pm j}\right)_{\infty}\left(p \omega_{2 n+1}^{ \pm 2 j} ; p^{2}\right)_{\infty} \prod_{1 \leqslant i<j \leqslant n}\left(p \omega_{2 n+1}^{j-i}, p \omega_{2 n+1}^{i-j}, p \omega_{2 n+1}^{i+j}, p \omega_{2 n+1}^{-i-j}\right)_{\infty}}, \\
Q_{D_{n}} & =\frac{2 \prod_{j=1}^{n} \theta\left(-p^{j-1} ; p^{2 n-2}\right)}{\prod_{1 \leqslant i<j \leqslant n}\left(p \omega_{2 n-2}^{j-i}, p \omega_{2 n-2}^{i-j}, p \omega_{2 n-2}^{i+j-2}, p \omega_{2 n-2}^{2-i-j}\right)_{\infty}} .
\end{aligned}
$$

It remains to simplify these expressions into the form given in Proposition 6.1. We indicate a way to organize the computations for $R=B_{n}$; the other cases can be treated similarly. We factor $Q_{B_{n}}$ as $F_{1} / F_{2} F_{3}$, where

$$
\begin{aligned}
& F_{1}=\prod_{j=1}^{n}\left(-p^{j-1} ; p^{2 n-1}\right)_{\infty}\left(-p^{2 n-j} ; p^{2 n-1}\right)_{\infty}, \\
& F_{2}=\prod_{j=1}^{n}\left(-p \omega_{2 n-1}^{j-n}\right)_{\infty}\left(-p \omega_{2 n-1}^{n-j}\right)_{\infty}, \\
& F_{3}=\prod_{1 \leqslant i<j \leqslant n}\left(p \omega_{2 n-1}^{j-i}, p \omega_{2 n-1}^{i-j}, p \omega_{2 n-1}^{i+j-1}, p \omega_{2 n-1}^{1-i-j}\right)_{\infty} .
\end{aligned}
$$

In $F_{1}$, we make the change of variables $j \mapsto 2 n+1-j$ in the second factor and use (2.1) to obtain

$$
F_{1}=\prod_{j=1}^{2 n}\left(-p^{j-1} ; p^{2 n-1}\right)_{\infty}=2(-p ; p)_{\infty}\left(-p^{2 n-1} ; p^{2 n-1}\right)_{\infty}
$$




\section{ELLiPtic DETERMinANT EVALUATIONS AND MACDONALD IDENTITIES}

Similarly, in $F_{2}$ we change $j \mapsto 2 n-j$ in the second factor, obtaining

$$
F_{2}=\prod_{j=1}^{n}\left(-p \omega_{2 n-1}^{j-n}\right)_{\infty} \prod_{j=n}^{2 n-1}\left(-p \omega_{2 n-1}^{j-n}\right)_{\infty}=(-p ; p)_{\infty}\left(-p^{2 n-1} ; p^{2 n-1}\right)_{\infty} .
$$

Finally, in $F_{3}$ we rewrite the first two factors as

$$
\frac{1}{(p)_{\infty}^{n}} \prod_{i, j=1}^{n}\left(p \omega_{2 n-1}^{j-i}\right)_{\infty}
$$

Making the change of variables $i \mapsto 2 n-i$, this equals

$$
\frac{1}{(p)_{\infty}^{n}} \prod_{i=n}^{2 n-1} \prod_{j=1}^{n}\left(p \omega_{2 n-1}^{i+j-1}\right)_{\infty}
$$

In the fourth factor in $F_{3}$, we change $(i, j) \mapsto(n-i, n+1-j)$, which gives

$$
\prod_{1 \leqslant i<j \leqslant n}\left(p \omega_{2 n-1}^{1-i-j}\right)_{\infty}=\prod_{1 \leqslant j \leqslant i \leqslant n-1}\left(p \omega_{2 n-1}^{i+j-1}\right)_{\infty} .
$$

Thus, the third and fourth factor can be combined into

$$
\prod_{i=1}^{n-1} \prod_{j=1}^{n}\left(p \omega_{2 n-1}^{j+i-1}\right)_{\infty}
$$

which, together with (6.2), gives

$$
F_{3}=\frac{1}{(p)_{\infty}^{n}} \prod_{j=1}^{n} \prod_{i=1}^{2 n-1}\left(p \omega_{2 n-1}^{j+i-1}\right)_{\infty}=\frac{1}{(p)_{\infty}^{n}} \prod_{j=1}^{n}\left(p^{2 n-1} ; p^{2 n-1}\right)_{\infty}=\frac{\left(p^{2 n-1} ; p^{2 n-1}\right)_{\infty}^{n}}{(p ; p)_{\infty}^{n}} .
$$

In conclusion, this shows that

$$
Q_{B_{n}}=\frac{2(p ; p)_{\infty}^{n}}{\left(p^{2 n-1} ; p^{2 n-1}\right)_{\infty}^{n}}
$$

in agreement with Proposition 6.1.

The determinant evaluations in Proposition 6.1 imply the following multiple Laurent expansions. We give two versions of each identity, the second being obtained from the first by an application of one of the classical Weyl denominator formulas (1.2). To verify that these identities agree with Macdonald's, the easiest way is to take the second version, replace $p$ by $q, m_{i}$ by $-m_{i}$ and $x_{i}$ by $x_{i}^{-1}$, and then compare with how the Macdonald identities are written in [Sta89]. (Equation (3.16) in [Sta89] should $\operatorname{read} c(q)=1 /(q)_{\infty}^{n}$, not $c(q)=q /(q)_{\infty}^{n}$.)

Corollary 6.2. The following identities hold:

$$
\begin{aligned}
(p ; p)_{\infty}^{n-1} W_{A_{n-1}}(x) & =\sum_{\substack{m_{1}, \ldots, m_{n} \in \mathbb{Z} \\
m_{1}+\cdots+m_{n}=0}} \sum_{\sigma \in S_{n}} \operatorname{sgn}(\sigma) \prod_{i=1}^{n} x_{i}^{n m_{i}+\sigma(i)-1} p^{n\left(\begin{array}{c}
m_{i} \\
2
\end{array}\right)+(\sigma(i)-1) m_{i}} \\
& =\sum_{\substack{m_{1}, \ldots, m_{n} \in \mathbb{Z} \\
m_{1}+\cdots+m_{n}=0}} \prod_{i=1}^{n} x_{i}^{n m_{i}} p^{n\left(\begin{array}{c}
m_{i} \\
2
\end{array}\right)} \prod_{1 \leqslant i<j \leqslant n}\left(x_{j} p^{m_{j}}-x_{i} p^{m_{i}}\right),
\end{aligned}
$$


H. Rosengren And M. Schlosser

$$
\begin{aligned}
& (p ; p)_{\infty}^{n} W_{B_{n}}(x)=\sum_{\substack{m_{1}, \ldots, m_{n} \in \mathbb{Z} \\
m_{1}+\cdots+m_{n} \equiv 0(2)}} \sum_{\sigma \in S_{n}} \operatorname{sgn}(\sigma) \prod_{i=1}^{n} x_{i}^{(2 n-1) m_{i}} p^{(2 n-1)\left(\begin{array}{c}
m_{i} \\
2
\end{array}\right)+(n-1) m_{i}} \\
& \times\left(\left(x_{i} p^{m_{i}}\right)^{\sigma(i)-n}-\left(x_{i} p^{m_{i}}\right)^{n+1-\sigma(i)}\right) \\
& =\sum_{\substack{m_{1}, \ldots, m_{n} \in \mathbb{Z} \\
m_{1}+\cdots+m_{n} \equiv 0(2)}} \prod_{i=1}^{n} x_{i}^{(2 n-1) m_{i}+1-n} p^{(2 n-1)\left(\begin{array}{c}
m_{i} \\
2
\end{array}\right)} \prod_{i=1}^{n}\left(1-x_{i} p^{m_{i}}\right) \\
& \times \prod_{1 \leqslant i<j \leqslant n}\left(x_{j} p^{m_{j}}-x_{i} p^{m_{i}}\right)\left(1-x_{i} x_{j} p^{m_{i}+m_{j}}\right), \\
& \left(p^{2} ; p^{2}\right)_{\infty}(p ; p)_{\infty}^{n-1} W_{B_{n}^{\vee}}(x)=\sum_{\substack{m_{1}, \ldots, m_{n} \in \mathbb{Z} \\
m_{1}+\cdots+m_{n} \equiv 0(2)}} \sum_{\sigma \in S_{n}} \operatorname{sgn}(\sigma) \prod_{i=1}^{n} x_{i}^{2 n m_{i}} p^{2 n\left(\begin{array}{c}
m_{i} \\
2
\end{array}\right)+n m_{i}} \\
& \times\left(\left(x_{i} p^{m_{i}}\right)^{\sigma(i)-n-1}-\left(x_{i} p^{m_{i}}\right)^{n+1-\sigma(i)}\right) \\
& =\sum_{\substack{m_{1}, \ldots, m_{n} \in \mathbb{Z} \\
m_{1}+\cdots+m_{n} \equiv 0(2)}} \prod_{i=1}^{n} x_{i}^{n\left(2 m_{i}-1\right)} p^{2 n\left(\begin{array}{c}
m_{i} \\
2
\end{array}\right)} \prod_{i=1}^{n}\left(1-x_{i}^{2} p^{2 m_{i}}\right) \\
& \times \prod_{1 \leqslant i<j \leqslant n}\left(x_{j} p^{m_{j}}-x_{i} p^{m_{i}}\right)\left(1-x_{i} x_{j} p^{m_{i}+m_{j}}\right), \\
& (p ; p)_{\infty}^{n} W_{C_{n}}(x)=\sum_{m_{1}, \ldots, m_{n} \in \mathbb{Z}} \sum_{\sigma \in S_{n}} \operatorname{sgn}(\sigma) \prod_{i=1}^{n} x_{i}^{(2 n+2) m_{i}} p^{(2 n+2)\left(\begin{array}{c}
m_{i} \\
2
\end{array}\right)+(n+1) m_{i}} \\
& \times\left(\left(x_{i} p^{m_{i}}\right)^{\sigma(i)-n-1}-\left(x_{i} p^{m_{i}}\right)^{n+1-\sigma(i)}\right) \\
& =\sum_{m_{1}, \ldots, m_{n} \in \mathbb{Z}} \prod_{i=1}^{n} x_{i}^{(2 n+2) m_{i}-n} p^{(2 n+2)\left(\begin{array}{c}
m_{i} \\
2
\end{array}\right)+m_{i}} \prod_{i=1}^{n}\left(1-x_{i}^{2} p^{2 m_{i}}\right) \\
& \times \prod_{1 \leqslant i<j \leqslant n}\left(x_{j} p^{m_{j}}-x_{i} p^{m_{i}}\right)\left(1-x_{i} x_{j} p^{m_{i}+m_{j}}\right), \\
& \left(p^{\frac{1}{2}} ; p^{\frac{1}{2}}\right)_{\infty}(p ; p)_{\infty}^{n-1} W_{C_{n}^{\vee}}(x)=\sum_{m_{1}, \ldots, m_{n} \in \mathbb{Z}} \sum_{\sigma \in S_{n}} \operatorname{sgn}(\sigma) \prod_{i=1}^{n} x_{i}^{2 n m_{i}} p^{2 n\left(\begin{array}{c}
m_{i} \\
2
\end{array}\right)+\left(n-\frac{1}{2}\right) m_{i}} \\
& \times\left(\left(x_{i} p^{m_{i}}\right)^{\sigma(i)-n}-\left(x_{i} p^{m_{i}}\right)^{n+1-\sigma(i)}\right) \\
& =\sum_{m_{1}, \ldots, m_{n} \in \mathbb{Z}} \prod_{i=1}^{n} x_{i}^{2 n m_{i}+1-n} p^{2 n\left(\begin{array}{c}
m_{i} \\
2
\end{array}\right)+\frac{1}{2} m_{i}} \prod_{i=1}^{n}\left(1-x_{i} p^{m_{i}}\right) \\
& \times \prod_{1 \leqslant i<j \leqslant n}\left(x_{j} p^{m_{j}}-x_{i} p^{m_{i}}\right)\left(1-x_{i} x_{j} p^{m_{i}+m_{j}}\right), \\
& (p ; p)_{\infty}^{n} W_{B C_{n}}(x)=\sum_{m_{1}, \ldots, m_{n} \in \mathbb{Z}} \sum_{\sigma \in S_{n}} \operatorname{sgn}(\sigma) \prod_{i=1}^{n} x_{i}^{(2 n+1) m_{i}} p^{(2 n+1)\left(\begin{array}{c}
m_{i} \\
2
\end{array}\right)+n m_{i}} \\
& \times\left(\left(x_{i} p^{m_{i}}\right)^{\sigma(i)-n}-\left(x_{i} p^{m_{i}}\right)^{n+1-\sigma(i)}\right) \\
& =\sum_{m_{1}, \ldots, m_{n} \in \mathbb{Z}} \prod_{i=1}^{n} x_{i}^{(2 n+1) m_{i}+1-n} p^{(2 n+1)\left(\begin{array}{c}
m_{i} \\
2
\end{array}\right)+m_{i}} \prod_{i=1}^{n}\left(1-x_{i} p^{m_{i}}\right) \\
& \times \prod_{1 \leqslant i<j \leqslant n}\left(x_{j} p^{m_{j}}-x_{i} p^{m_{i}}\right)\left(1-x_{i} x_{j} p^{m_{i}+m_{j}}\right),
\end{aligned}
$$


Elliptic Determinant EVAluations And Macdonald identities

$$
\begin{aligned}
(p ; p)_{\infty}^{n} W_{D_{n}}(x)= & \frac{1}{2} \sum_{\substack{m_{1}, \ldots, m_{n} \in \mathbb{Z} \\
m_{1}+\cdots+m_{n} \equiv 0(2)}} \sum_{\sigma \in S_{n}} \operatorname{sgn}(\sigma) \prod_{i=1}^{n} x_{i}^{(2 n-2) m_{i}} p^{(2 n-2)\left(\begin{array}{c}
m_{i} \\
2
\end{array}\right)+(n-1) m_{i}} \\
& \times\left(\left(x_{i} p^{m_{i}}\right)^{\sigma(i)-n}+\left(x_{i} p^{m_{i}}\right)^{n-\sigma(i)}\right) \\
= & \sum_{\substack{m_{1}, \ldots, m_{n} \in \mathbb{Z} \\
m_{1}+\cdots+m_{n} \equiv 0(2)}} \prod_{i=1}^{n} x_{i}^{(n-1)\left(2 m_{i}-1\right)} p^{(2 n-2)\left(\begin{array}{c}
m_{i} \\
2
\end{array}\right)} \\
& \times \prod_{1 \leqslant i<j \leqslant n}\left(x_{j} p^{m_{j}}-x_{i} p^{m_{i}}\right)\left(1-x_{i} x_{j} p^{m_{i}+m_{j}}\right), \quad n \geqslant 2 .
\end{aligned}
$$

Proof. We start from the determinant evaluations in Proposition 6.1. In the cases when there are two theta functions in each matrix elements (i.e. $\left.R \neq A_{n-1}\right)$, we apply $\theta\left(x ; p^{N}\right)=\theta\left(p^{N} / x ; p^{N}\right)$ to the second theta function. We then expand the left-hand sides using (2.2). For $C_{n}, C_{n}^{\vee}$ and $B C_{n}$, this leads immediately to the desired expansions.

For $A_{n-1}$, expanding also the factor $\theta\left(t x_{1} \cdots x_{n}\right)$, we obtain

$$
\begin{gathered}
\sum_{m_{1}, \ldots, m_{n}=-\infty}^{\infty} \sum_{\sigma \in S_{n}} \operatorname{sgn}(\sigma) \prod_{i=1}^{n}(-1)^{n m_{i}} p^{n\left(\begin{array}{c}
m_{i} \\
2
\end{array}\right)+(\sigma(i)-1) m_{i}} t^{m_{i}} x_{i}^{n m_{i}+\sigma(i)-1} \\
=(p)_{\infty}^{n-1} W_{A_{n-1}}(x) \sum_{N=-\infty}^{\infty}(-1)^{N} p^{\left(\begin{array}{c}
N \\
2
\end{array}\right)\left(t x_{1} \cdots x_{n}\right)^{N}}
\end{gathered}
$$

Viewing this as a Laurent series in $t$, taking the constant term gives the desired result. (Picking out any other Laurent coefficient gives an equivalent identity.)

For $B_{n}, B_{n}^{\vee}$ and $D_{n}$, we obtain series with the correct terms but different range of summation. More precisely, we find that

$$
2 X=\sum_{m_{1}, \ldots, m_{n} \in \mathbb{Z}} f\left(m_{1}, \ldots, m_{n}\right),
$$

where the identity we wish to prove is

$$
X=\sum_{\substack{m_{1}, \ldots, m_{n} \in \mathbb{Z} \\ m_{1}+\cdots+m_{n} \equiv 0(2)}}(-1)^{m_{1}+\cdots+m_{n}} f\left(m_{1}, \ldots, m_{n}\right)
$$

in the cases $B_{n}$ and $B_{n}^{\vee}$, and

$$
X=\sum_{\substack{m_{1}, \ldots, m_{n} \in \mathbb{Z} \\ m_{1}+\cdots+m_{n} \equiv 0(2)}} f\left(m_{1}, \ldots, m_{n}\right)
$$

in the case of $D_{n}$. In any case, it remains to show that

$$
\sum_{\substack{m_{1}, \ldots, m_{n} \in \mathbb{Z} \\ m_{1}+\cdots+m_{n} \equiv 0(2)}} f\left(m_{1}, \ldots, m_{n}\right)=\sum_{\substack{m_{1}, \ldots, m_{n} \in \mathbb{Z} \\ m_{1}+\cdots+m_{n} \equiv 1(2)}} f\left(m_{1}, \ldots, m_{n}\right) .
$$

To see this, we fix $\sigma$ and restrict attention to the index $m_{i}$, where $i=\sigma^{-1}(1)$. Then, we may write $f\left(m_{1}, \ldots, m_{n}\right)=C\left(g\left(m_{i}\right)+g\left(m_{i}+1\right)\right)$, where $C$ is independent of $m_{i}$ and

$$
\begin{aligned}
& g(m)=(-1)^{m} p^{(2 n-1)\left(\begin{array}{c}
m \\
2
\end{array}\right)} x_{i}^{(2 n-1) m+1-n}, \quad R=B_{n}, \\
& g(m)=(-1)^{m} p^{2 n\left(\begin{array}{c}
m \\
2
\end{array}\right)} x_{i}^{n(2 m-1)}, \quad R=B_{n}^{\vee}, \\
& g(m)=p^{(2 n-2)\left(\begin{array}{c}
m \\
2
\end{array}\right)} x_{i}^{(n-1)(2 m-1)}, \quad R=D_{n} .
\end{aligned}
$$

This observation completes the proof. 


\section{H. Rosengren And M. Schlosser}

\section{ACKNOWLEDGEMENTS}

We thank Eric Rains for his comments on Corollary 4.10, leading to some improvements in that part of the paper, and Vitaly Tarasov for clarifying how to obtain Corollary 4.5 from the results of [TV97], see Remark 4.6.

\section{REFERENCES}

DS01 J. F. van Diejen and V. P. Spiridonov, Elliptic Selberg integrals, Internat. Math. Res. Notices 20 (2001), 1083-1110.

Dys72 F. Dyson, Missed opportunities, Bull. Amer. Math. Soc. 78 (1972), 635-652.

FK99 H. M. Farkas and I. Kra, On the quintuple product identity, Proc. Amer. Math. Soc. 127 (1999), 771-778.

Fay73 J. D. Fay, Theta functions on Riemann surfaces, Lecture Notes in Mathematics, vol. 352 (Springer, Berlin, 1973).

Fro82 F. G. Frobenius, Über die elliptischen Funktionen zweiter Art, J. reine angew. Math. 93 (1882), $53-68$.

FS77 F. G. Frobenius and L. Stickelberger, Zur Theorie der elliptischen Functionen, J. reine angew. Math. 83 (1877), 175-179.

GK97 R. A. Gustafson and C. Krattenthaler, Determinant evaluations and $U(n)$ extensions of Heine's ${ }_{2} \phi_{1}$-transformations, in Special functions, q-series and related topics, eds M. E. H. Ismail, D. R. Masson and M. Rahman (American Mathematical Society, Providence, RI, 1997), 83-90.

Has97 K. Hasegawa, Ruijsenaars' commuting difference operators as commuting transfer matrices, Comm. Math. Phys. 187 (1997), 289-325.

Hir83 M. D. Hirschhorn, A simple proof of an identity of Ramanujan, J. Aust. Math. Soc. Ser. A 34 (1983), 31-35.

Hir00 M. D. Hirschhorn, An identity of Ramanujan, and applications, in q-Series from a contemporary perspective, eds M. E. H. Ismail and D. W. Stanton, Contemporary Mathematics, vol. 254 (American Mathematical Society, Providence, RI, 2000), 229-234.

Kac90 V. Kac, Infinite-dimensional Lie algebras, 3rd edn (Cambridge University Press, Cambridge, 1990).

KN03 Y. Kajihara and M. Noumi, Multiple elliptic hypergeometric series. An approach from the Cauchy determinant, Indag. Math. (N.S.) 14 (2003), 395-421.

Kra95 C. Krattenthaler, The major counting of nonintersecting lattice paths and generating functions for tableaux, Mem. Amer. Math. Soc. 115 (1995), no. 552.

Kra99 C. Krattenthaler, Advanced determinant calculus, Sém. Lothar. Combin. 42 (1999), B42q.

Kra05 C. Krattenthaler, Advanced determinant calculus: a complement, Linear Algebra Appl. 411 (2005), 68-166.

Mac72 I. G. Macdonald, Affine root systems and Dedekind's $\eta$-function, Invent. Math. 15 (1972), 91-143.

Oka04 S. Okada, An elliptic generalization of Schur's Pfaffian identity, Adv. Math., to appear.

Rai03 E. M. Rains, Transformations of elliptic hypergeometric integrals, Preprint (2003), math.QA/0309252.

Rai05 E. M. Rains, Recurrences for elliptic hypergeometric integrals, in Elliptic integrable systems, eds M. Noumi and K. Takasaki, Rokko Lectures in Mathematics, vol. 18 (Kobe University, 2005), 183-199.

Ros01 H. Rosengren, A proof of a multivariable elliptic summation formula conjectured by Warnaar, in q-Series with applications to combinatorics, number theory, and physics, eds B. C. Berndt and K. Ono, Contemporary Mathematics, vol. 291 (American Mathematical Society, Providence, RI, 2001), 193-202.

Ros04 H. Rosengren, Elliptic hypergeometric series on root systems, Adv. Math. 181 (2004), 417-447. 


\section{Elliptic DETERMinANT EVALUATIONS AND MACDONALD IDENTITIES}

Ros05 H. Rosengren, Sums of triangular numbers from the Frobenius determinant, Preprint (2005), math.NT/0504272.

RS03 H. Rosengren and M. Schlosser, Summations and transformations for multiple basic and elliptic hypergeometric series by determinant evaluations, Indag. Math. (N.S.) 14 (2003), 483-514.

Rui87 S. N. M. Ruijsenaars, Complete integrability of relativistic Calogero-Moser systems and elliptic function identities, Comm. Math. Phys. 110 (1987), 191-213.

Sch97 M. Schlosser, Multidimensional matrix inversions and $A_{r}$ and $D_{r}$ basic hypergeometric series, Ramanujan J. 1 (1997), 243-274.

Sch99 M. Schlosser, Some new applications of matrix inversions in $A_{r}$, Ramanujan J. 3 (1999), 405-461.

Sch00a M. Schlosser, A new multidimensional matrix inversion in $A_{r}$, in q-Series from a contemporary perspective, eds M. E. H. Ismail and D. W. Stanton, Contemporary Mathematics, vol. 254 (American Mathematical Society, Providence, RI, 2000), 413-432.

Sch00b M. Schlosser, Summation theorems for multidimensional basic hypergeometric series by determinant evaluations, Discrete Math. 210 (2000), 151-169.

Spi03 V. Spiridonov, Theta hypergeometric integrals, Algebra i Analiz 15 (2003), 161-215.

Sta89 D. Stanton, An elementary approach to the Macdonald identities, in q-Series and partitions, ed. D. Stanton, IMA Volumes in Mathematics and its Applications, vol. 18 (Springer, New York, 1989), 139-149.

Syl51 J. J. Sylvester, On the relation between the minor determinants of linearly equivalent quadratic functions, Philosophical Magazine 1 (1851), 295-305.

TV97 V. Tarasov and A. Varchenko, Geometry of q-hypergeometric functions, quantum affine algebras and elliptic quantum groups, Astérisque 246 (1997).

War02 S. O. Warnaar, Summation and transformation formulas for elliptic hypergeometric series, Constr. Approx. 18 (2002), 479-502.

Wat29 G. N. Watson, Theorems stated by Ramanujan. VII: Theorems on continued fractions, J. London Math. Soc. 4 (1929), 39-48.

Web91 H. Weber, Elliptische Functionen und algebraische Zahlen (Vieweg \& Sohn, Braunschweig, 1891).

WW96 E. T. Whittaker and G. N. Watson, A course of modern analysis, reprint of the 1927 fourth edition (Cambridge University Press, Cambridge, 1996).

Win69 L. Winquist, An elementary proof of $p(11 m+6) \equiv 0(\bmod 11)$, J. Combin. Theory 6 (1969), 56-59.

Hjalmar Rosengren hjalmar@math.chalmers.se

Department of Mathematical Sciences, Chalmers University of Technology and Göteborg University, SE-412 96 Göteborg, Sweden

Michael Schlosser schlosse@ap.univie.ac.at

Fakultät für Mathematik der Universität Wien, Nordbergstraße 15, A-1090 Wien, Austria 